J Org Chem. 2016 September 2; 81(17): 8035-8042. doi:10.1021/acs.joc.6b01421.

\title{
Alkylation of Sulfonamides with Trichloroacetimidates Under Thermal Conditions
}

\author{
Daniel R. Wallach and John D. Chisholm ${ }^{\star}$ \\ Department of Chemistry, 1-014 Center for Science and Technology, Syracuse University, \\ Syracuse, NY 13244
}

\section{Abstract \\ An intermolecular alkylation of sulfonamides with trichloroacetimidates is reported. This transformation does not require an exogenous acid, base, or transition metal catalyst, instead the addition occurs in refluxing toluene without additives. The sulfonamide alkylation partner appears to be only limited by sterics, with unsubstituted sulfonamides providing better yields than more encumbered $N$-alkyl sulfonamides. The trichloroacetimidate alkylating agent must be a stable cation precursor for the substitution reaction to proceed under these conditions.}

\section{Graphical abstract}

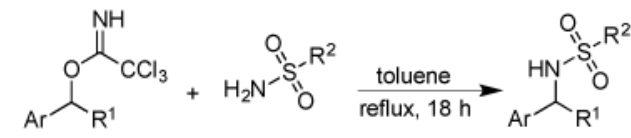

The sulfonamide functional group has played an important role in the development of numerous pharmaceuticals. While best known as antibiotics, sulfonamide scaffolds provide a diverse range of biological activity including antitumor, antiviral, diuretic, antiinflammatory and anti-hypertensive properties. ${ }^{1}$ Summaries of the top selling pharmaceuticals clearly demonstrate that sulfonamides are well represented in these valuable structures. ${ }^{2}$ Data on recently approved pharmaceuticals indicate that sulfonamides continue to be popular in drug discovery, ${ }^{3}$ with recent publications in the medicinal chemistry field corroborating that the investigation of sulfonamide-containing structures is ongoing. ${ }^{4}$ Sulfonamides also have proven useful in agricultural and insecticidal applications. ${ }^{5}$

In addition, sulfonamides serve an important function in synthetic organic chemistry, as they facilitate the introduction of nitrogen into organic molecules. The sulfonamide may then serve as a protecting group for the nitrogen atom during further synthetic manipulation. The use of sulfonamides as protecting groups in complex molecules was hindered by the harsh conditions ${ }^{6}$ needed to remove the sulfonamide. The development of 2-(trimethylsilyl)-

*Corresponding Author jdchisho@syr.edu.

Notes

The authors declare no competing financial interest.

Supporting Information Available: Copies of ${ }^{1} \mathrm{H}$ NMR and ${ }^{13} \mathrm{C}$ NMR spectra of new compounds and chiral HPLC data for compounds $\mathbf{2}$ and $\mathbf{3}$. This material is available free of charge via the Internet at http:// pubs.acs.org. 
ethanesulfonamide (SES-NH ${ }_{2}$ ) by Weinreb ${ }^{7}$ and the implementation of 2- and 4nitrobenzenesulfonamides by Fukuyama ${ }^{8}$ have resulted in a greatly increased use of sulfonamides in organic synthesis, primarily because these sulfonamides are readily and reliably removed under mild conditions. These developments have greatly popularized the use of sulfonamides in complex molecule synthesis.

With the obvious value of sulfonamides in chemical and pharmaceutical research, methods for their formation and elaboration have been heavily investigated. Classically substituted sulfonamides may be formed from amines and sulfonyl chlorides, ${ }^{9}$ by direct alkylation of sulfonamides with alkyl halides, ${ }^{10}$ or through reductive amination. ${ }^{11}$ The Mitsunobu reaction has also been employed to convert an alcohol into a sulfonamide. ${ }^{7 b, 8 a, 12}$ Given the high level of interest in sulfonamides, it is perhaps unsurprising that new, more atomeconomical catalytic methods for their formation have continued to evolve. Many of these methods employ transition metal catalysts, including the hydroaminations of alkenes, ${ }^{13} \mathrm{C}-\mathrm{H}$ activation methods,,${ }^{14}$ metal catalyzed additions to $\mathrm{N}$-sulfonyl imines, ${ }^{6 \mathrm{~b}, 15}$ alkylation via $\pi$ allyl metal complexes, ${ }^{16}$ and alkylation of alcohols via borrowing hydrogen methods. ${ }^{17}$ Direct alkylation of benzylic and allylic alcohols and ethers has also been explored, ${ }^{18}$ although these methods typically require the use of strong Brønsted or Lewis acids and elevated temperatures.

As part of our recent investigations into the use of trichloroacetimidates to alkylate carboxylic acids, alcohols, thiols and anilines under mild conditions, ${ }^{19}$ we sought to determine if sulfonamides could be similarly elaborated. Lewis acid promoted alkylation with trichloroacetimidates has been widely utilized in the synthesis of ethers ${ }^{20}$ and carbohydrates. ${ }^{21}$ Trichloroacetimidate displacements produce only trichloroacetamide as a byproduct, which is typically unreactive and may be removed by washing with aqueous $\mathrm{NaOH}$ solution. ${ }^{19 \mathrm{~d}}$ More recently there have been several reports of additive-free trichloroacetimidate substitution reactions, with only heating being necessary to effect the displacement of some trichloroacetimidates with alcohols ${ }^{19 \mathrm{c}}$ and thiols. ${ }^{19 \mathrm{~d}}$ Development of a new, catalyst-free method to access substituted sulfonamides would provide an inexpensive method to access these structures under mild conditions. Little is known about the reactivity of sulfonamides with trichloroacetimidates, although recently Kuroda and co-workers reported an intramolecular $\mathrm{S}_{\mathrm{N}} 2$ ' substitution reaction of allylic imidates and sulfonamides catalyzed by a chiral phosphoric acid. ${ }^{22}$ To our knowledge this is the only example of a reaction between a trichloroacetimidate and a sulfonamide in the literature.

Initially, the reaction of $p$-toluenesulfonamide $\mathbf{1}$ with 1-phenethyl trichloroacetimidate $\mathbf{2}$ was studied (Table 1). Utilizing $\mathrm{BF}_{3} \cdot \mathrm{OEt}_{2}$ as a catalyst in toluene, alkylation was rapid but only a $29 \%$ yield of the desired alkylation product 3 was realized. This poor yield was due to a significant portion of imidate $\mathbf{2}$ rearranging to the corresponding trichloroacetamide $\mathbf{4}$ during the alkylation. This rearrangement also complicated the isolation of the product $\mathbf{3}$, as the acetamide possessed similar chromatographic motility. Brønsted acid catalysis had proven superior in our study on the alkylation of anilines with trichloroacetimidates, ${ }^{19 \mathrm{e}}$ and so these catalysts were investigated next. Weak acids like PPTS gave a slower reaction resulting in a $50 \%$ yield, while stronger acids like dinitrobenzenesulfonic acid (DNBSA) gave a more rapid alkylation in $71 \%$ yield. While the reaction may be catalyzed with Brønsted or Lewis 
acids, given the acidity of the sulfonamide functional group the transformation in principle may also occur without a catalyst under thermal conditions as observed with alcohols and thiols. ${ }^{19 \mathrm{c}, 19 \mathrm{~d}}$

Heating a mixture of sulfonamide $\mathbf{1}$ and imidate $\mathbf{2}$ in toluene to reflux for 18 hours (entry 4) resulted in a $76 \%$ yield of alkylated product. Shorter reaction times were not as effective, as the reaction was incomplete and therefore provided more moderate yields. Attempts to perform the alkylation at lower temperatures in toluene (entry 7) and in lower boiling solvents like THF (entry 8) provided only unreacted starting materials, so a temperature near refluxing toluene was necessary for the alkylation to proceed. At this point it was noted that while $p$-toluenesulfonamide $\mathbf{1}$ was only slightly soluble in refluxing toluene, imidate $\mathbf{2}$ and the trichloroacetamide byproduct $\mathbf{5}$ were completely soluble, which led to the hypothesis that as trichloroacetamide $\mathbf{5}$ was formed it could compete with the sulfonamide $\mathbf{1}$ as a nucleophile, leading to the undesired product $\mathbf{4}$. To combat this problem the imidate $\mathbf{2}$ was added to the reaction mixture in portions which resulted in an increased yield of $86 \%$ (entry 9). Even though 1.5 equivalents of imidate was used this reaction, no increase in yield was noted with 1.5 equiv of imidate without the portionwise addition (entry 10). As entry 9 provided the best yield, this procedure was used henceforth.

The scope of the reaction of with respect to sulfonamide was then investigated (Table 2). A variety of different sulfonamides were found to undergo the alkylation reaction with imidate 2 in useful yields. Benzenesulfonamides substituted with electron donating groups provided excellent yields alkylated sulfonamide products (entries 1-3). 2-Nitrobenzenesulfonamide 12 only provided a yield of $13 \%$ (entry 5), which was disappointing as Fukuyama has demonstrated the utility of this sulfonamide for installing amines. ${ }^{8 \mathrm{a}}$ This poor yield was attributed to the particular insolubility of this sulfonamide in toluene. Use of other solvents (a,a,a-trifluorotoluene, DCE, acetonitrile) did not improve the yield of the transformation. After some experimentation a solution was found, with a reaction catalyzed by $\mathrm{BF}_{3}$ ? $\mathrm{OEt}_{2}$ providing a 70\% yield when the imidate added over the course of one hour via syringe pump to the reaction mixture.

Alkyl sulfonamides were also well tolerated in the reaction with yields ranging from 70 to 79\% (entries 6-8). The successful alkylation of 2-(trimethylsilyl)-ethanesulfonamide $\mathbf{1 8}$ is particularly notable, as this sulfonamide can be removed to reveal the corresponding amine by treatment with fluoride. Sulfonamide $\mathbf{1 8}$ is often utilized to introduce a protected nitrogen into complex molecules. ${ }^{7 \mathrm{c}}$ The inexpensive carboxylic sulfonamide saccharin 20 reacted with trichloroacetimidate 2 with excellent yield of $98 \%$ (entry 9). Saccharin has been used as a replacement for phthalimide in the Gabriel synthesis, ${ }^{23}$ making this an inexpensive method to incorporate nitrogen in organic substrates. $\mathrm{N}$-alkyl sulfonamides gave lower yields, with N-methylbenzenesulfonamide 22 providing $27 \%$ of $\mathbf{2 3}$ (entry 10). NBenzylbenzenesulfonamide 24 failed to react (entry 11). The decreased reactivity of Nsubstituted sulfonamides can be attributed to sterics, and explains why no dialkylation products were observed.

To further probe the reaction scope the reactivity of differentially substituted trichloroacetimidates with $p$-toluenesulfonamide 1 was investigated (Table 3). Secondary 
benzylic trichloroacetimidates were effective substrates, providing the $\mathrm{N}$-alkyl sulfonamides in good yields. These reactions tolerated a sterically encumbering ortho-substituent on the aromatic ring next to the reactive center (as seen in the formation of $\mathbf{3 3}$ and $\mathbf{3 5}$ ). The furanyl imidate $\mathbf{3 6}$ proved to be susceptible to hydrolysis and rearrangement to the corresponding acetamide, but still provided a $44 \%$ yield of substitution product 37 .

Diphenylmethyl imidate readily participated in the reaction to provide an $89 \%$ yield of sulfonamide 39. This is notable as diphenylmethyl groups have been utilized as protecting groups for sulfonamides. ${ }^{24}$ Other diarylmethyl imidates also provided synthetically useful yields in the alkylation reaction, providing $\mathbf{4 1}$ in $67 \%$ yield. A number of primary benzylic trichloroacetimidates were also evaluated. Use of 4-methoxybenzyl trichloroacetimidate $\mathbf{4 2}$ or $\boldsymbol{o}$-tolyl trichloroacetimidate $\mathbf{4 4}$ provided the alkylated products $\mathbf{4 3}$ and $\mathbf{4 5}$, but the yields were lower than was observed for the secondary benzylic imidates. Benzyl trichloroacetimidate $\mathbf{4 6}$ was unreactive under these conditions. Secondary allylic trichloroacetimidates provided good yields of alkylated products, as seen in the case of compound 49. Simple allyl trichloroacetimidate $\mathbf{5 0}$ provided a much lower yield of the product. Methyl and tert-butyl trichloroacetimidate were also evaluated, but product was only observed in the methyl case which gave low conversion.

As defined in Table 3, imidates that are precursors to stable carbocations provide higher yields under these reaction conditions. This implies that the reaction proceeds through an $\mathrm{S}_{\mathrm{N}} 1$ pathway, where the imidate ionizes and then is trapped with the sulfonamide. To further test this hypothesis the enantiopure imidate $(R)-2^{19}$ e was subjected to the reaction conditions, providing the sulfonamide 3 in $81 \%$ yield (Scheme 1), however the product was racemic. This result is inconsistent with an $\mathrm{S}_{\mathrm{N}} 2$ pathway, and further supports an $\mathrm{S}_{\mathrm{N}} 1$ pathway as is commonly invoked for trichloroacetimidate substitution reactions with benzylic substrates.

The new reaction was then applied to the synthesis of an interesting ketoprofen analog $\mathbf{5 8}$ (Scheme 2) discovered by Sakurai and co-workers. ${ }^{25}$ Sulfonamide $\mathbf{5 8}$ showed a number of interesting pharmacological properties such as $\mathrm{LTD}_{4}$ antagonistic activity, $\mathrm{TXA}_{2}$ antagonistic activity, and $\mathrm{TXA}_{2}$ synthase inhibitory activity. ${ }^{25-26}$ The synthetic scheme used by Sakurai to synthesize $\mathbf{5 8}$ involved the forming the azide, which was then reduced to the corresponding amine followed by transformation into the sulfonamide. Alternatively, sulfonamide $\mathbf{5 8}$ could be synthesized via imidate $\mathbf{4 0}$, which avoids the use of the toxic sodium azide and eliminating the need for azide reduction. Initially, alcohol $\mathbf{5 6}$ was synthesized following the procedure of Sakurai and coworkers (Scheme 2) ${ }^{25}$ Formation of the corresponding imidate $\mathbf{4 0}$ proceeded in near quantitative yield. Imidate $\mathbf{4 0}$ was displaced with 4-chlorobenzenesulfonamide $\mathbf{1 0}$ to obtain sulfonamide $\mathbf{5 7}$ in $88 \%$ yield. Sulfonamide $\mathbf{5 7}$ may be transformed to ketoprofen analog $\mathbf{5 8}$ via saponification. ${ }^{25}$

In summary, thermal conditions for the alkylation of sulfonamides with trichloroacetimidates have been developed. Unsubstituted sulfonamides were found to undergo alkylation well, but $\mathrm{N}$-substituted sulfonamides were not effectively alkylated. The trichloroacetimidate alkylation partner must be a precursor to a stabilized carbocation, with the preponderance of evidence supporting an $S_{N} 1$ reaction pathway in most cases. The 
formal synthesis of a biologically active ketoprofen analog was accomplished using this new substitution reaction.

\section{Experimental Section}

\section{Representative Sulfonamide Substitution Procedure A}

To a flame dried round bottom flask under an atmosphere of argon was added $p$-toluene sulfonamide $1(0.13 \mathrm{~g}, 0.77 \mathrm{mmol})$ and toluene $(4 \mathrm{~mL})$. Phenethyl imidate $\mathbf{2}^{19 \mathrm{e}}(51 \mathrm{mg}, 0.19$ $\mathrm{mmol}$ ) was added and the reaction mixture was heated to reflux. Phenethyl imidate $\mathbf{2}(0.05$ $\mathrm{g}, 0.19 \mathrm{mmol}$ ) was added to the refluxing reaction mixture every 30 minutes until $1.14 \mathrm{mmol}$ (1.5 equiv) of phenethyl imidate was added. After stirring at reflux overnight, the reaction mixture was allowed to cool to room temperature, preadsorbed on silica gel and purified by silica gel chromatography (30\% ethyl acetate/70\% hexanes) to give $0.180 \mathrm{~g}(86 \%)$ of substituted sulfonamide $\mathbf{3}$ as a white solid.

\section{4-Methyl-N-(1-phenylethyl)benzenesulfonamide (3). ${ }^{27}$}

Prepared using procedure A $(0.180 \mathrm{~g}, 86 \%)$ using the known imidate ${ }^{19 \mathrm{e}}$ and purified using silica gel chromatography (30\% ethyl acetate/70\% hexanes).

3. White solid $(0.18 \mathrm{~g}, 86 \%)$; $\mathrm{mp}=74-78{ }^{\circ} \mathrm{C}$; $\mathrm{TLC}_{\mathrm{f}}=0.43$ (20\% ethyl acetate $/ 80 \%$ hexanes); ${ }^{1} \mathrm{H}$ NMR $\left(300 \mathrm{MHz}, \mathrm{CDCl}_{3}\right) \delta 7.61(\mathrm{dt}, J=8.7,2.1 \mathrm{~Hz}, 2 \mathrm{H}), 7.21-7.17(\mathrm{~m}, 5 \mathrm{H})$, 7.11-7.08 (m, 2H), $4.76(\mathrm{~d}, J=6.8 \mathrm{~Hz}, 1 \mathrm{H}), 4.47$ (p, $J=6.9 \mathrm{~Hz}, 1 \mathrm{H}), 2.39$ (s, 3H), 1.42 (d, $J$ $=6.9 \mathrm{~Hz}, 3 \mathrm{H}) ;{ }^{13} \mathrm{C} \mathrm{NMR}\left(100 \mathrm{MHz}, \mathrm{CDCl}_{3}\right) \delta 143.1,142.0,137.7,129.4,128.5,127.5$, 127.1, 126.1, 53.6, 23.5, 21.5. Chiral HPLC analysis: Chiralcel OD (heptane/i-PrOH = $\left.90 / 10,1.0 \mathrm{~mL} / \mathrm{min}, 254 \mathrm{~nm}, 25^{\circ} \mathrm{C}\right): \mathrm{t}=10.6,12.8 \mathrm{~min}$.

\section{4-Methoxy-N-(1-phenylethyl)benzenesulfonamide (7). ${ }^{28}$}

Prepared using procedure $\mathbf{A}(0.17 \mathrm{~g}, 75 \%)$ using the known imidate ${ }^{19 \mathrm{e}}$ and purified by silica gel chromatography (100\% DCM).

7. Waxy off-white solid $(0.17 \mathrm{~g}, 75 \%)$; $\mathrm{mp}=94-96{ }^{\circ} \mathrm{C}$; $\operatorname{TLC} \mathrm{R}_{\mathrm{f}}=0.25(100 \% \mathrm{DCM}) ;{ }^{1} \mathrm{H}$ NMR (400 MHz, $\left.\mathrm{CDCl}_{3}\right) \delta 7.65(\mathrm{dt}, J=9.2,2.8 \mathrm{~Hz}, 2 \mathrm{H}), 7.21-7.15(\mathrm{~m}, 3 \mathrm{H}), 7.11-7.09(\mathrm{~m}$, 2H), $6.82(\mathrm{dt}, J=9.6,2.8 \mathrm{~Hz}, 2 \mathrm{H}), 5.35(\mathrm{~d}, J=7.2 \mathrm{~Hz}, 1 \mathrm{H}), 4.43(\mathrm{p}, J=7.2 \mathrm{~Hz}, 1 \mathrm{H}), 3.82$ (s, 3H), $1.40(\mathrm{~d}, J=7.2 \mathrm{~Hz}, 3 \mathrm{H}) ;{ }^{13} \mathrm{C}$ NMR $\left(100 \mathrm{MHz}, \mathrm{CDCl}_{3}\right) \delta 162.6,142.2,132.2,129.2$, $128.5,127.4,126.2,114.0 .55 .6,53.7,23.6$.

\section{N-(1-Phenylethyl)benzenesulfonamide (9). ${ }^{29}$}

Prepared using procedure A $(0.14 \mathrm{~g}, 72 \%)$ using the known imidate ${ }^{19 \mathrm{e}}$ and purified using silica gel chromatography (30\% ethyl acetate/70\% hexanes).

9. White solid ( $0.14 \mathrm{~g}, 85 \%) ; \mathrm{mp}=87-91{ }^{\circ} \mathrm{C}$; $\mathrm{TLC}_{\mathrm{f}}=0.44$ (30\% ethyl acetate $/ 70 \%$ hexanes); ${ }^{1} \mathrm{H}$ NMR $\left(400 \mathrm{MHz}, \mathrm{CDCl}_{3}\right) \delta$ 7.74-7.71 (m, 2H), $7.44(\mathrm{td}, J=6.4,1.2 \mathrm{~Hz}, 1 \mathrm{H})$, 7.36-7.31 (m, 2H), 7.14-7.07 (m, 5H), $5.65(\mathrm{~d}, J=7.2 \mathrm{~Hz}, 1 \mathrm{H}), 4.48(\mathrm{p}, J=6.8 \mathrm{~Hz}, 1 \mathrm{H})$, $1.40(\mathrm{~d}, J=7.2 \mathrm{~Hz}, 3 \mathrm{H}) ;{ }^{13} \mathrm{C}$ NMR $\left(100 \mathrm{MHz}, \mathrm{CDCl}_{3}\right) \delta 142.0,140.7,132.3,128.8,128.5$, 127.4, 127.0, 126.1, 53.8, 23.6. 


\section{4-Chloro-N-(1-phenylethyl)benzenesulfonamide (11). ${ }^{30}$}

Prepared using procedure A $(0.19 \mathrm{~g}, 83 \%)$ using the known imidate ${ }^{19 \mathrm{e}}$ and purified using silica gel chromatography (10\% ethyl acetate/90\% hexanes).

11. White amorphous solid $(0.15 \mathrm{~g}, 67 \%) ; \mathrm{mp}=71-75^{\circ} \mathrm{C}$; TLC $\mathrm{R}_{\mathrm{f}}=0.21$ (10\% ethyl acetate/90\% hexanes); ${ }^{1} \mathrm{H}$ NMR $\left(400 \mathrm{MHz}, \mathrm{CDCl}_{3}\right) \delta 7.60(\mathrm{~d}, J=8.4 \mathrm{~Hz}, 2 \mathrm{H}), 7.30$ (d, $J=$ $8.8 \mathrm{~Hz}, 3 \mathrm{H}), 7.19-7.17(\mathrm{~m}, 2 \mathrm{H}), 7.08-7.05(\mathrm{~m}, 2 \mathrm{H}), 5.14$ (d, $J=7.2 \mathrm{~Hz}, 1 \mathrm{H}), 4.50$ (p, $J=6.8$ $\mathrm{Hz}, 1 \mathrm{H}), 1.44(\mathrm{~d}, J=6.8 \mathrm{~Hz}, 3 \mathrm{H}) ;{ }^{13} \mathrm{C}$ NMR $\left(100 \mathrm{MHz}, \mathrm{CDCl}_{3}\right) \delta 141.6,139.2,138.7$, 129.0, 128.6, 128.5, 127.6, 126.1, 53.9, 23.6.

\section{2-Nitro-N-(1-phenylethyl)benzenesulfonamide (13). ${ }^{31}$}

2-Nitrobenzenesulfonamide $(0.18 \mathrm{~g}, 0.87 \mathrm{mmol})$ and $\mathrm{BF}_{3} \cdot \mathrm{OEt}_{2}(0.02 \mathrm{~g}, 0.09 \mathrm{mmol})$ were suspended in DCM (4 mL). The suspension was heated to reflux. A $0.1 \mathrm{M}$ solution of 1phenethyl trichloroacetimidate $2^{19 \mathrm{e}}(0.30 \mathrm{~g}, 1.13 \mathrm{mmol})$ in DCM was added to the suspension using a syringe pump over the course of one hour. The reaction was refluxed for 18h. After cooling to room temperature, the reaction was poured into saturated aq. $\mathrm{NaHCO}_{3}$ and extracted with DCM (3x). The combined organic extracts were dried over $\mathrm{Na}_{2} \mathrm{SO}_{4}$, filtered and concentrated in vacuo. The residue was purified via silica gel chromatography (100\% DCM) providing $\mathbf{1 3}(0.18 \mathrm{~g}, 70 \%)$ as a white solid. The sulfonamide $\mathbf{1 3}$ was also prepared using procedure $\mathbf{A}(0.04 \mathrm{~g}, 13 \%)$.

13. White solid ( $0.18 \mathrm{~g}, 70 \%) ; \mathrm{mp}=89-91{ }^{\circ} \mathrm{C}$; $\mathrm{TLC} \mathrm{R}_{\mathrm{f}}=0.58(100 \% \mathrm{DCM}) ;{ }^{1} \mathrm{H}$ NMR $(400$ $\left.\mathrm{MHz}, \mathrm{CDCl}_{3}\right) \delta 7.71(\mathrm{dd}, J=8.0,1.2 \mathrm{~Hz}, 1 \mathrm{H}), 7.67(\mathrm{dd}, J=8.0,1.6 \mathrm{~Hz}, 1 \mathrm{H}), 7.54(\mathrm{td}, J=$ 7.6, 1.2 Hz, 1H), 7.40 (td, $J=8.0,1.6 \mathrm{~Hz}, 1 \mathrm{H}), 7.12-7.06(\mathrm{~m}, 5 \mathrm{H}), 5.77(\mathrm{~d}, J=8.4 \mathrm{~Hz}, 1 \mathrm{H})$, $4.69(\mathrm{p}, J=6.8 \mathrm{~Hz}, 1 \mathrm{H}), 1.52(\mathrm{~d}, J=7.2 \mathrm{~Hz}, 3 \mathrm{H}) ;{ }^{13} \mathrm{C} \mathrm{NMR}\left(100 \mathrm{MHz}, \mathrm{CDCl}_{3}\right) \delta 147.3$, 141.2, 134.5, 132.9, 132.5, 130.8, 128.5, 127.7, 126.1, 124.9, 55.0, 23.7.

\section{$\mathrm{N}$-(1-Phenylethyl)methanesulfonamide (15). ${ }^{31}$}

Prepared using procedure A $(0.12 \mathrm{~g}, 79 \%)$ using the known imidate ${ }^{19 \mathrm{e}}$ and purified using silica gel chromatography (30\% ethyl acetate/70\% hexanes).

15. Yellow oil ( $0.12 \mathrm{~g}, 79 \%)$; $\mathrm{TLC} \mathrm{R}_{\mathrm{f}}=0.35$ (30\% ethyl acetate $/ 70 \%$ hexanes); ${ }^{1} \mathrm{H}$ NMR $\left(400 \mathrm{MHz}, \mathrm{CDCl}_{3}\right) \delta 7.39-7.26(\mathrm{~m}, 5 \mathrm{H}), 5.16(\mathrm{~d}, J=7.2 \mathrm{~Hz}, 1 \mathrm{H}) ; 4.64(\mathrm{p}, J=7.2 \mathrm{~Hz}, 1 \mathrm{H})$, $2.61(\mathrm{~s}, 3 \mathrm{H}), 1.53(\mathrm{~d}, J=6.8 \mathrm{~Hz}, 3 \mathrm{H}) ;{ }^{13} \mathrm{C} \mathrm{NMR}\left(100 \mathrm{MHz}, \mathrm{CDCl}_{3}\right) \delta 142.4,129.0,128.0$, $126.2,53.8,41.8,24.0$.

\section{$\mathrm{N}$-(1-Phenylethyl)ethanesulfonamide (17). ${ }^{32}$}

Prepared using procedure A $(0.15 \mathrm{~g}, 76 \%)$ using the known imidate ${ }^{19 \mathrm{e}}$ and purified using silica gel chromatography (100\% DCM flushed with 30\% ethyl acetate/70\% hexanes).

17. White solid $(0.15 \mathrm{~g}, 76 \%) ; \mathrm{mp}=89-91{ }^{\circ} \mathrm{C}$; $\mathrm{TLC} \mathrm{R}_{\mathrm{f}}=0.34$ (30\% ethyl acetate $/ 70 \%$ hexanes); ${ }^{1} \mathrm{H}$ NMR (400 MHz, $\mathrm{CDCl}_{3}$ ) $\delta$ 7.36-7.26 (m, 5H), 5.17 (br d, $\left.J=7.1 \mathrm{~Hz}, 1 \mathrm{H}\right)$, 4.62 (p, $J=7.1 \mathrm{~Hz}, 1 \mathrm{H}), 2.76$ (h, $J=7.4 \mathrm{~Hz}, 1 \mathrm{H}), 2.61$ (h, $J=7.4 \mathrm{~Hz}, 1 \mathrm{H}), 1.54$ (d, $J=6.9$ $\mathrm{Hz}, 3 \mathrm{H}), 1.17$ (t, $J=7.4 \mathrm{~Hz}, 3 \mathrm{H}) ;{ }^{13} \mathrm{C}$ NMR $\left(100 \mathrm{MHz}, \mathrm{CDCl}_{3}\right) \delta 142.8,128.8,127.9$, 126.2, 53.7, 47.9, 24.2, 8.0. 


\section{N-(1-Phenylethyl)-2-(trimethylsilyl)ethanesulfonamide (19). ${ }^{33}$}

Prepared using procedure $\mathbf{A}(0.13 \mathrm{~g}, 70 \%)$ using the known imidate ${ }^{19 \mathrm{e}}$ and purified by silica gel chromatography (30\% ethyl acetate/80\% hexanes).

19. White crystals $(0.13 \mathrm{~g}, 70 \%) ; \mathrm{mp}=61-64{ }^{\circ} \mathrm{C}$; $\mathrm{TLC} \mathrm{R}_{\mathrm{f}}=0.58$ (20\% ethyl acetate $/ 80 \%$ hexanes); ${ }^{1} \mathrm{H}$ NMR (400 MHz, $\left.\mathrm{CDCl}_{3}\right) \delta$ 7.38-7.27 (m, 5H), 4.73 (d, $\left.J=6.9 \mathrm{~Hz}, 1 \mathrm{H}\right), 4.62$ (p, $J=6.9 \mathrm{~Hz}, 1 \mathrm{H}), 2.61(\mathrm{td}, J=14.0,3.9 \mathrm{~Hz}, 1 \mathrm{H}), 2.47(\mathrm{td}, J=13.9,4.4 \mathrm{~Hz}, 1 \mathrm{H}), 1.54(\mathrm{~d}, J$ $=6.9 \mathrm{~Hz}, 3 \mathrm{H}), 0.86(\mathrm{td}, J=13.8,4.0,1 \mathrm{H}), 0.74(\mathrm{td}, J=14.0,4.3 \mathrm{~Hz}, 1 \mathrm{H}),-0.13(\mathrm{~s}, 9 \mathrm{H}) ;{ }^{13} \mathrm{C}$ NMR (100 MHz, $\left.\mathrm{CDCl}_{3}\right) \delta$ 143.0, 129.1, 128.2, 126.4, 53.9, 50.0, 24.3, 10.5, -2.0.

\section{3-Oxo-N-(1-phenylethyl)benzo[d]isothiazole-2(3H)-sulfonamide 1,1-dioxide (21). ${ }^{34}$}

Prepared using procedure $\mathbf{A}$ using the known imidate ${ }^{19 \mathrm{e}}$ and purified by silica gel chromatography (20\% ethyl acetate/ $80 \%$ hexanes). The crude product was then taken up in ethyl acetate $(30 \mathrm{~mL})$ and washed with $2 \mathrm{M} \mathrm{NaOH}(5 \times 20 \mathrm{~mL})$. The organic layers were combined, dried over sodium sulfate and concentrated in vacuo to provide $\mathbf{2 1}$ as a clear colorless oil $(0.22 \mathrm{~g}, 98 \%)$.

21. Clear colorless oil $(0.22 \mathrm{~g}, 98 \%)$; $\mathrm{TLC} \mathrm{R}_{\mathrm{f}}=0.37$ (20\% ethyl acetate/80\% hexanes); ${ }^{1} \mathrm{H}$ NMR $\left(400 \mathrm{MHz}, \mathrm{CDCl}_{3}\right) \delta$ 7.98-7.95 (m, 1H), 7.90-7.88 (m, 1H), $7.82(\mathrm{td}, J=7.4,1.2 \mathrm{~Hz}$, $1 \mathrm{H}), 7.77$ (td, $J=7.2,1.4 \mathrm{~Hz}, 1 \mathrm{H}), 7.60-7.57(\mathrm{~m}, 2 \mathrm{H}), 7.36$ (tt, $J=6.8,1.2 \mathrm{~Hz}, 2 \mathrm{H}), 7.29$ (tt, $J=6.2,1.3 \mathrm{~Hz}, 1 \mathrm{H}), 5.45(\mathrm{q}, J=7.3 \mathrm{~Hz}, 1 \mathrm{H}), 2.03(\mathrm{~d}, J=7.3 \mathrm{~Hz}, 3 \mathrm{H}) ;{ }^{13} \mathrm{C}$ NMR $(100 \mathrm{MHz}$, $\left.\mathrm{CDCl}_{3}\right) \delta 158.5,138.6,137.8,134.6,134.2,128.5,128.2,127.7,127.3,125.0,120.7,53.0$, 17.7.

\section{4-Methyl-N-(1-phenylethyl)benzenesulfonamide (23). ${ }^{35}$}

Prepared using procedure A $(0.06 \mathrm{~g}, 27 \%)$ using the known imidate ${ }^{19 \mathrm{e}}$ and purified using silica gel chromatography (100\% DCM).

23. White powder $(0.06 \mathrm{~g}, 27 \%)$; $\mathrm{mp}=60-62{ }^{\circ} \mathrm{C}$; $\mathrm{TLC} \mathrm{R}_{\mathrm{f}}=0.52(100 \% \mathrm{DCM}) ;{ }^{1} \mathrm{H}$ NMR $\left(400 \mathrm{MHz}, \mathrm{CDCl}_{3}\right) \delta 7.74(\mathrm{dt}, J=8.4,1.8 \mathrm{~Hz}, 2 \mathrm{H}), 7.32-7.24(\mathrm{~m}, 7 \mathrm{H}), 5.29(\mathrm{q}, J=7.0 \mathrm{~Hz}$, $1 \mathrm{H}), 2.57$ (s, 3H), $2.43(\mathrm{~s}, 3 \mathrm{H}), 1.29(\mathrm{~d}, J=7.0 \mathrm{~Hz}, 3 \mathrm{H}) ;{ }^{13} \mathrm{C} \mathrm{NMR}\left(100 \mathrm{MHz}, \mathrm{CDCl}_{3}\right) \delta$ $143.1,139.9,137.3,129.7,128.4,127.5,127.3,127.1,54.8,28.4,21.5,15.2$.

\section{1-(p-Tolyl)ethyl 2,2,2-trichloroacetimidate (26)}

To a round bottom flask under argon was added 1-(p-tolyl)ethanol $(0.51 \mathrm{~g}, 3.74 \mathrm{mmol})$, trichloroacetonitrile $(0.48 \mathrm{~mL}, 4.86 \mathrm{mmol})$ and DCM $(7 \mathrm{~mL})$. DBU $(0.06 \mathrm{~mL}, 0.37 \mathrm{mmol})$ was added and the reaction mixture was stirred at room temperature for $18 \mathrm{~h}$. Triethylamine $(1 \mathrm{~mL})$ was added to the reaction mixture and the solvent was removed in vacuo. Purification of the residue by silica gel chromatography (1\% triethylamine/9\% ethyl acetate/90\% hexanes) provided imidate $\mathbf{2 6}$ as white crystals (0.81 g, 77\%).

26. White crystals $(0.81 \mathrm{~g}, 77 \%)$; $\mathrm{mp}=41-42{ }^{\circ} \mathrm{C}$; $\mathrm{TLC} \mathrm{R}_{\mathrm{f}}=0.50$ (10\% ethyl acetate $/ 90 \%$ hexanes); IR (KBr) 3344, 2982, 2931, 2868, 1663, $1285 \mathrm{~cm}^{-1} ;{ }^{1} \mathrm{H} \mathrm{NMR} \mathrm{(400} \mathrm{MHz,} \mathrm{CDCl}_{3}$ ) $\delta 8.27(\mathrm{br} \mathrm{s}, 1 \mathrm{H}), 7.31(\mathrm{~d}, J=8.2 \mathrm{~Hz}, 2 \mathrm{H}), 7.17(\mathrm{~d}, J=7.8 \mathrm{~Hz}, 2 \mathrm{H}), 5.94(\mathrm{q}, J=6.6 \mathrm{~Hz}, 1 \mathrm{H})$, $2.34(\mathrm{~s}, 3 \mathrm{H}), 1.63(\mathrm{~d}, J=6.6 \mathrm{~Hz}, 3 \mathrm{H}) ;{ }^{13} \mathrm{C} \mathrm{NMR}\left(100 \mathrm{MHz}, \mathrm{CDCl}_{3}\right) \delta 161.7,138.4,137.6$, 
129.2, 125.8, 91.8, 77.2, 22.2, 21.2. Anal. Calcd for $\mathrm{C}_{11} \mathrm{H}_{12} \mathrm{Cl}_{3} \mathrm{NO}$ : C, 47.09; $\mathrm{H}, 4.31$; , 4.99. Found: C,46.75; H, 4.05; N, 4.80.

\section{4-Methyl-N-(1-( $p$-tolyl)ethyl)benzenesulfonamide (27). ${ }^{36}$}

Prepared using procedure A $(0.19 \mathrm{~g}, 89 \%)$ using imidate $\mathbf{2 6}$ and purified using silica gel chromatography (30\% ethyl acetate/70\% hexanes).

27. White powder $(0.19 \mathrm{~g}, 89 \%) ; \mathrm{mp}=118-119^{\circ} \mathrm{C}$; $\mathrm{TLC} \mathrm{R}_{\mathrm{f}}=0.65(30 \%$ ethyl acetate $/ 70 \%$ hexanes); ${ }^{1} \mathrm{H}$ NMR (400 MHz, $\left.\mathrm{CDCl}_{3}\right) \delta 7.62(\mathrm{dt}, J=8.3,1.0 \mathrm{~Hz}, 2 \mathrm{H}), 7.17(\mathrm{dd}, J=8.5,0.6$ Hz, 2H), 6.98 (app s, 4H), 5.04 (d, J=7.0 Hz, 1H), 4.40 (p, J=6.9 Hz, 1H), 2.38 (s, 3H), 2.27 (s, 3H) 1.39 (d, $J=6.8 \mathrm{~Hz}, 3 \mathrm{H}) ;{ }^{13} \mathrm{C} \mathrm{NMR}\left(100 \mathrm{MHz}, \mathrm{CDCl}_{3}\right) \delta 143.0,139.1,137.8$, 137.1, 129.4, 129.2, 127.1, 126.1, 53.4, 23.5, 21.5, 21.0.

\section{1,2,3,4-Tetrahydronaphthalen-1-yl 2,2,2-trichloroacetimidate (28)}

To a round bottom flask under argon was added 1,2,3,4-tetrahydronaphthalen-1-ol (1.00 g, $6.75 \mathrm{mmol})$, DBU $(0.10 \mathrm{~mL}, 0.67 \mathrm{mmol})$ and DCM $(23 \mathrm{~mL})$. The reaction mixture was stirred at room temperature for 15 minutes and then cooled to $0^{\circ} \mathrm{C}$ in an ice/water bath. Trichloroacetonitrile $(0.88 \mathrm{~mL}, 8.77 \mathrm{mmol})$ was added and the reaction mixture was warmed to room temperature and stirred overnight. The solvent was then removed in vacuo.

Triethylamine $(1 \mathrm{~mL})$ was added and the residue was purified by silica gel chromatography (2\% triethylamine/10\% ethyl acetate/88\% hexanes) to provide $\mathbf{2 8}$ as a clear colorless oil $(1.68 \mathrm{~g}, 94 \%)$.

28. Clear colorless oil (1.68 g, 94\%); $\mathrm{TLC} \mathrm{R}_{\mathrm{f}}=$ dec. (10\% ethyl acetate/90\% hexanes); IR (thin film on KBr) 3341, 3064, 3024, 2940, 2869, $1657 \mathrm{~cm}^{-1} ;{ }^{1} \mathrm{H}$ NMR (400 MHz, $\mathrm{CDCl}_{3}$ ) $\delta 8.39$ (br s, 1H), 7.39-7.37 (m, 1H), 7.27-7.14 (m, 3H), $6.10(\mathrm{t}, J=4.8 \mathrm{~Hz}, 1 \mathrm{H}), 2.93-2.74$ $(\mathrm{m}, 2 \mathrm{H}), 2.22-1.96(\mathrm{~m}, 3 \mathrm{H}), 1.89-1.81(\mathrm{~m}, 1 \mathrm{H}) ;{ }^{13} \mathrm{C} \mathrm{NMR}\left(100 \mathrm{MHz}, \mathrm{CDCl}_{3}\right) \delta 162.5$, 138.1, 134.2, 129.6, 129.1, 128.3, 126.1, 92.1, 75.5, 29.1, 27.9, 19.1. Anal. Calcd for $\mathrm{C}_{12} \mathrm{H}_{12} \mathrm{Cl}_{3} \mathrm{NO}$ : C, 49.26; H, 4.13; N,4.75. Found: C,48.92; H, 4.44; N, 4.92.

\section{4-Methyl-N-(1,2,3,4-tetrahydronaphthalen-1-yl)benzenesulfonamide (29). ${ }^{37}$}

Prepared using procedure A $(0.18 \mathrm{~g}, 79 \%)$ using imidate $\mathbf{2 8}$ and purified using silica gel chromatography (30\% ethyl acetate/70\% hexanes).

29. Beige solid $(0.18 \mathrm{~g}, 79 \%) ; \mathrm{mp}=115-118{ }^{\circ} \mathrm{C}$; $\mathrm{TLC} \mathrm{R}_{\mathrm{f}}=0.62(30 \%$ ethyl acetate $/ 70 \%$ hexanes); ${ }^{1} \mathrm{H}$ NMR (400 MHz, $\left.\mathrm{CDCl}_{3}\right) \delta 7.75(\mathrm{td}, J=8.4,1.9 \mathrm{~Hz}, 2 \mathrm{H}), 7.26(\mathrm{~d}, J=7.9 \mathrm{~Hz}$, 2H), 7.05 (td, $J=7.3,1.2 \mathrm{~Hz}, 1 \mathrm{H}), 7.00-6.94(\mathrm{~m}, 2 \mathrm{H}), 6.87$ (d, $J=7.6 \mathrm{~Hz}, 1 \mathrm{H}), 4.67$ (br d, $J$ $=7.8 \mathrm{~Hz}, 1 \mathrm{H}), 4.37(\mathrm{p}, J=5.2 \mathrm{~Hz}, 1 \mathrm{H}), 2.71-2.54(\mathrm{~m}, 2 \mathrm{H}), 2.38(\mathrm{~s}, 3 \mathrm{H}), 1.79-1.71(\mathrm{~m}, 3 \mathrm{H})$, 1.66-1.62 (m, 1H); $\left.{ }^{13} \mathrm{C} \mathrm{NMR} \mathrm{(100} \mathrm{MHz,} \mathrm{CDCl}_{3}\right) \delta$ 143.4, 138.2, 137.6, 135.6, 129.8, 129.2, $128.8,127.6,127.2,126.3,51.9,30.8,28.9,21.6,19.2$.

\section{1-(Naphthalen-1-yl)ethyl 2,2,2-trichloroacetimidate (30)}

To a round bottom flask under argon was added 1-(naphthalen-1-yl)ethanol (0.85 g, 4.92 $\mathrm{mmol})$, trichloroacetonitrile $(0.59 \mathrm{~mL}, 5.90 \mathrm{mmol})$ and DCM $(12 \mathrm{~mL})$. DBU $(0.08 \mathrm{~mL}, 0.49$ $\mathrm{mmol}$ ) was added to the reaction mixture and the reaction mixture was stirred at room 
temperature for $18 \mathrm{~h}$. Triethylamine $(1 \mathrm{~mL})$ was added to the reaction mixture and the reaction mixture was purified by silica gel chromatography (2\% triethylamine/8\% ethyl acetate/90\% hexanes) to provide $\mathbf{3 0}$ as a clear colorless oil (1.32 $\mathrm{g}, 85 \%)$.

30. Clear colorless oil ( $1.32 \mathrm{~g}, 85 \%)$; TLC $\mathrm{R}_{\mathrm{f}}=0.80$ (10\% ethyl acetate/90\% hexanes); IR (thin film on KBr) 3339, 3052, 2983, 2933, 2870, 1661, $1598 \mathrm{~cm}^{-1} ;{ }^{1} \mathrm{H}$ NMR (400 MHz, $\left.\mathrm{CDCl}_{3}\right) \delta 8.36(\mathrm{br} \mathrm{s}, 1 \mathrm{H}), 8.11(\mathrm{~d}, J=8.5 \mathrm{~Hz}, 1 \mathrm{H}), 7.89-7.87(\mathrm{~m}, 1 \mathrm{H}), 7.81(\mathrm{~d}, J=8.2 \mathrm{~Hz}$, $1 \mathrm{H}), 7.71(\mathrm{~d}, J=7.1 \mathrm{~Hz}, 1 \mathrm{H}), 7.56-7.47(\mathrm{~m}, 3 \mathrm{H}), 6.74(\mathrm{q}, J=6.6 \mathrm{~Hz}, 1 \mathrm{H}), 1.81(\mathrm{~d}, J=6.6$ $\mathrm{Hz}, 3 \mathrm{H}) ;{ }^{13} \mathrm{C}$ NMR $\left(100 \mathrm{MHz}, \mathrm{CDCl}_{3}\right) \delta 161.7,137.1,133.8,130.2,128.9,128.5,126.3$, 125.7, 125.4, 123.2, 123.0, 91.8, 74.6, 21.34. Anal. Calcd for $\mathrm{C}_{14} \mathrm{H}_{12} \mathrm{Cl}_{3} \mathrm{NO}: \mathrm{C}, 53.11 ; \mathrm{H}$, 3.82; N,4.42. Found: C,53.47; H, 3.62; N, 4.75 .

\section{4-Methyl-N-(1-(naphthalen-1-yl)ethyl)benzenesulfonamide (31). ${ }^{28}$}

Prepared using procedure A $(0.20 \mathrm{~g}, 79 \%)$ using imidate $\mathbf{3 0}$ and purified using silica gel chromatography (30\% ethyl acetate/70\% hexanes).

31. Orange oil $(0.20 \mathrm{~g}, 79 \%)$; $\mathrm{TLC} \mathrm{R}_{\mathrm{f}}=0.43$ (30\% ethyl acetate. $70 \%$ hexanes); ${ }^{1} \mathrm{H}$ NMR $\left(400 \mathrm{MHz}, \mathrm{CDCl}_{3}\right) \delta$ 7.89-7.87 (m, 1H), 7.80-7.78 (m, 1H), $7.67(\mathrm{~d}, J=8.1 \mathrm{~Hz}, 1 \mathrm{H}), 7.56$ $(\mathrm{dt}, J=8.3,1.7 \mathrm{~Hz}, 2 \mathrm{H}), 7.47-7.40(\mathrm{~m}, 2 \mathrm{H}), 7.36(\mathrm{dd}, J=7.2,1.0 \mathrm{~Hz}, 1 \mathrm{H}), 7.31-7.27(\mathrm{~m}$, $1 \mathrm{H}), 7.04(\mathrm{~d}, J=7.9 \mathrm{~Hz}, 2 \mathrm{H}), 5.28(\mathrm{p}, J=6.8 \mathrm{~Hz}, 1 \mathrm{H}), 5.15(\mathrm{~d}, J=6.8 \mathrm{~Hz}, 1 \mathrm{H}), 2.31$ (s, 3H), $1.58(\mathrm{~d}, J=6.8 \mathrm{~Hz}, 3 \mathrm{H}) ;{ }^{13} \mathrm{C} \mathrm{NMR}\left(100 \mathrm{MHz}, \mathrm{CDCl}_{3}\right) \delta 143.1,137.7,137.5,133.8,130.1$, $129.3,128.8,128.1,127.1,126.3,125.6,125.3,123.4,122.6,49.8,23.2,21.4$.

\section{1-(o-Tolyl)ethyl 2,2,2-trichloroacetimidate (32)}

To a round bottom flask under argon was added 1-(o-tolyl)ethanol $(1.08 \mathrm{~g}, 8.89 \mathrm{mmol})$, trichloroacetonitrile $(1.16 \mathrm{~mL}, 11.56 \mathrm{mmol})$ and DCM $(17 \mathrm{~mL})$. DBU $(0.13 \mathrm{~mL}, 0.89 \mathrm{mmol})$ was added and the reaction mixture was stirred at room temperature for $18 \mathrm{~h}$. The solvent was then removed in vacuo. Triethylamine $(1 \mathrm{~mL})$ was added and the residue was purified by silica gel chromatography ( $1 \%$ triethylamine/9\% ethyl acetate/90\% hexanes) to provide 32 as white crystals $(1.82 \mathrm{~g}, 73 \%)$.

32. Clear colorless oil ( $1.82 \mathrm{~g}, 73 \%)$; $T L C \mathrm{R}_{\mathrm{f}}=0.68$ ( $10 \%$ ethyl acetate/ $90 \%$ hexanes); IR (KBr) 3342, 3025, 2980, 2931, 1662, $\left.1288 \mathrm{~cm}^{-1} ;{ }^{1} \mathrm{H} \mathrm{NMR} \mathrm{(400} \mathrm{MHz,} \mathrm{CDCl}_{3}\right) \delta 8.27$ (br s, $1 \mathrm{H}), 7.51-7.48(\mathrm{~m}, 1 \mathrm{H}), 7.25-7.14(\mathrm{~m}, 3 \mathrm{H}), 6.14(\mathrm{q}, J=6.5 \mathrm{~Hz}, 1 \mathrm{H}), 2.42(\mathrm{~s}, 3 \mathrm{H}), 1.61(\mathrm{~d}, J$ $=6.6 \mathrm{~Hz}, 3 \mathrm{H}) ;{ }^{13} \mathrm{C} \mathrm{NMR}\left(100 \mathrm{MHz}, \mathrm{CDCl}_{3}\right) \delta 161.5,139.7,134.7,130.3,127.7,126.3$, 125.1, 91.8, 74.3, 21.0, 19.0. Anal. Calcd for $\mathrm{C}_{11} \mathrm{H}_{12} \mathrm{Cl}_{3} \mathrm{NO}$ : C, 47.09; H, 4.31; N,4.99. Found: C, 46.88; H, 4.06; N, 4.81 .

\section{4-Methyl-N-(1-(o-tolyl)ethyl)benzenesulfonamide (33). ${ }^{6 \mathrm{~b}}$}

Prepared using procedure A $(0.21 \mathrm{~g}, 94 \%)$ using imidate 32 and purified using silica gel chromatography (30\% ethyl acetate/70\% hexanes).

33. Off-white solid $(0.31 \mathrm{~g}, 94 \%)$; $\mathrm{mp}=87-89^{\circ} \mathrm{C}$; $\mathrm{TLC} \mathrm{R}_{\mathrm{f}}=0.56$ (30\% ethyl acetate $/ 70 \%$ hexanes); ${ }^{1} \mathrm{H}$ NMR $\left(400 \mathrm{MHz}, \mathrm{CDCl}_{3}\right) \delta 7.50(\mathrm{dt}, J=8.3,1.7 \mathrm{~Hz}, 2 \mathrm{H}), 7.06-7.01(\mathrm{~m}, 3 \mathrm{H})$, 6.95-6.87 (m, 3H), $5.60(\mathrm{~d}, J=7.2 \mathrm{~Hz}, 1 \mathrm{H}), 4.62(\mathrm{p}, J=6.9 \mathrm{~Hz}, 1 \mathrm{H}), 2.24(\mathrm{~s}, 3 \mathrm{H}), 2.10(\mathrm{~s}$, 
$3 \mathrm{H}), 1.23(\mathrm{~d}, J=6.8 \mathrm{~Hz}, 3 \mathrm{H}) ;{ }^{13} \mathrm{C} \mathrm{NMR}\left(100 \mathrm{MHz}, \mathrm{CDCl}_{3}\right) \delta 143.0,140.5,137.7,134.3$, $130.3,129.4,127.1,127.0,126.4,125.5,49.8,23.121 .5,19.0$.

\section{1-(6-Bromobenzo[d][1,3]dioxol-5-yl)ethyl 2,2,2-trichloroacetimidate (34)}

To a round bottom flask under argon was added 1-(6-bromobenzo[ $d][1,3]$ dioxol-5yl)ethanol ${ }^{38}(2.90 \mathrm{~g}, 11.83 \mathrm{mmol})$, DBU $(0.18 \mathrm{~mL}, 1.18 \mathrm{mmol})$ and DCM $(39 \mathrm{~mL})$. The reaction mixture was stirred at room temperature for $15 \mathrm{~min}$. and then cooled to $0^{\circ} \mathrm{C}$ in an ice/water bath. Trichloroacetonitrile $(1.53 \mathrm{~mL}, 15.38 \mathrm{mmol})$ was added to the reaction mixture and the reaction mixture was warmed to room temperature and stirred overnight. The solvent was then removed in vacuo. Triethylamine $(1 \mathrm{~mL})$ was added and the residue was purified by silica gel chromatography (1\% triethylamine/50\% ethyl acetate/49\% hexanes) to provide $\mathbf{3 4}$ as a clear colorless oil (3.80 g, 83\%).

34. Clear colorless oil ( $3.80 \mathrm{~g}, 83 \%$ ); $\mathrm{TLC}_{\mathrm{f}}=0.69$ (30\% ethyl acetate $/ 70 \%$ hexanes); IR (thin film on $\mathrm{KBr}$ ) $3339,3080,2983,2930,2897,1667 \mathrm{~cm}^{-1} ;{ }^{1} \mathrm{H}$ NMR (400 MHz, $\mathrm{CDCl}_{3}$ ) $\delta 8.37(\mathrm{br} \mathrm{s}, 1 \mathrm{H}), 7.03(\mathrm{~s}, 1 \mathrm{H}), 6.98(\mathrm{~s}, 1 \mathrm{H}), 6.17(\mathrm{q}, J=6.4 \mathrm{~Hz}, 1 \mathrm{H}), 5.96(\mathrm{q}, J=1.2 \mathrm{~Hz}$, $2 \mathrm{H}), 1.58(\mathrm{~d}, J=6.4 \mathrm{~Hz}, 3 \mathrm{H}) ;{ }^{13} \mathrm{C} \mathrm{NMR}\left(100 \mathrm{MHz}, \mathrm{CDCl}_{3}\right) \delta 161.1,147.81,147.80,134.2$, 112.6, 112.0, 106.2, 101.8, 91.5, 76.3, 21.0. Anal. Calcd for $\mathrm{C}_{11} \mathrm{H}_{9} \mathrm{BrCl}_{3} \mathrm{NO}_{3}: \mathrm{C}, 33.92 ; \mathrm{H}$, 2.33; N,3.60. Found: C, 33.88; H, 2.49; N, 3.48.

\section{$\mathrm{N}$-(1-(6-Bromobenzo[d][1,3]dioxol-5-yl)ethyl)-4-methylbenzenesulfonamide (35)}

Prepared using procedure A $(0.29 \mathrm{~g}, 88 \%)$ using imidate $\mathbf{3 4}$ and purified by silica gel chromatography (30\% ethyl acetate. $70 \%$ hexanes).

35. Yellow powder $(0.29 \mathrm{~g}, 88 \%) ; \mathrm{mp}=106{ }^{\circ} \mathrm{C}(\mathrm{dec})$; $\mathrm{TLC} \mathrm{R}_{\mathrm{f}}=0.53$ (30\% ethyl acetate $/ 70 \%$ hexanes); IR (KBr) 3272, 2986, 1714, 1503, 1478, $1326 \mathrm{~cm}^{-1} ;{ }^{1} \mathrm{H}$ NMR (400 $\left.\mathrm{MHz}, \mathrm{CDCl}_{3}\right) \delta 7.64(\mathrm{dt}, J=8.5,1.9 \mathrm{~Hz}, 2 \mathrm{H}), 7.18(\mathrm{dd}, J=8.4,0.5 \mathrm{~Hz}, 2 \mathrm{H}), 6.82(\mathrm{~s}, 1 \mathrm{H})$, $6.69(\mathrm{~s}, 1 \mathrm{H}), 5.89(\mathrm{dd}, J=6.9,1.4 \mathrm{~Hz}, 2 \mathrm{H}), 5.46(\mathrm{~d}, J=6.6 \mathrm{~Hz}, 1 \mathrm{H}), 4.79(\mathrm{p}, J=6.8 \mathrm{~Hz}$, 1H), 2.38 (s, 3H), 1.32 (d, $J=6.8 \mathrm{~Hz}, 3 \mathrm{H}) ;{ }^{13} \mathrm{C} \mathrm{NMR}\left(100 \mathrm{MHz}, \mathrm{CDCl}_{3}\right) \delta$ 147.6, 147.4, 143.2, 137.0, 134.6, 129.4, 127.2, 112.37, 112.36, 107.4, 101.8, 52.9, 22.9, 21.5. Anal. Calcd for $\mathrm{C}_{16} \mathrm{H}_{16} \mathrm{BrNO}_{4} \mathrm{~S}: \mathrm{C}, 48.25 ; \mathrm{H}, 4.05 ; \mathrm{N}, 3.52$. Found: C, 48.07; H, 4.06; N, 3.30.

\section{1-(Furan-2-yl)pentyl 2,2,2-trichloroacetimidate (36)}

To a round bottom flask containing 1-(furan-2-yl)pentan-1-ol ${ }^{39}(0.52 \mathrm{~g}, 3.37 \mathrm{mmol})$ dissolved in DCM $(33 \mathrm{~mL})$ was added trichloroacetonitrile $(0.58 \mathrm{~g}, 4.04 \mathrm{mmol})$ and DBU $(0.05 \mathrm{~g}, 0.34 \mathrm{mmol})$. The reaction mixture was stirred at room temperature for 1 hour. The solvent was then removed in vacuo. Triethylamine $(1 \mathrm{~mL})$ was added and the residue was purified by silica gel chromatography (1\% triethylamine/19\% ethyl acetate/80\% hexanes) to provide $\mathbf{3 6}$ as a yellow oil $(0.32 \mathrm{~g}, 33 \%)$.

36. Yellow oil ( $0.32 \mathrm{~g}, 33 \%)$; TLC $\mathrm{R}_{\mathrm{f}}=0.62$ (20\% ethyl acetate/80\% hexanes); IR (KBr) 3346, 2960, 2873, 1656, $1501 \mathrm{~cm}^{-1} ;{ }^{1} \mathrm{H}$ NMR $\left(400 \mathrm{MHz}, \mathrm{CDCl}_{3}\right) \delta 8.35(\mathrm{~s}, 1 \mathrm{H}), 7.40(\mathrm{dd}, J$ $=1.8,0.8 \mathrm{~Hz}, 1 \mathrm{H}), 6.39(\mathrm{~d}, J=3.12 \mathrm{~Hz}, 1 \mathrm{H}), 6.34(\mathrm{dd}, J=3.2,1.8 \mathrm{~Hz}, 1 \mathrm{H}), 5.96(\mathrm{t}, J=6.6$ $\mathrm{Hz}, 1 \mathrm{H}), 2.18-2.08(\mathrm{~m}, 1 \mathrm{H}), 2.07-1.98(\mathrm{~m}, 1 \mathrm{H}), 1.47-1.26(\mathrm{~m}, 4 \mathrm{H}), 0.91(\mathrm{t}, J=6.9 \mathrm{~Hz}$, $3 \mathrm{H}) ;{ }^{13} \mathrm{C} \mathrm{NMR}\left(100 \mathrm{MHz}, \mathrm{CDCl}_{3}\right) \delta 162.0,152.3,142.4,110.2,108.5,91.7,73.9,32.4$, 
27.4, 22.3, 13.9. Anal. Calcd for $\mathrm{C}_{11} \mathrm{H}_{14} \mathrm{Cl}_{3} \mathrm{NO}_{2}$ : $\mathrm{C}, 44.25 ; \mathrm{H}, 4.73 ; \mathrm{N}, 4.69$. Found: $\mathrm{C}$, $44.49 ; \mathrm{H}, 4.45 ; \mathrm{N}, 4.79$.

\section{$\mathrm{N}$-(1-(Furan-2-yl)pentyl)-4-methylbenzenesulfonamide (37). ${ }^{40}$}

Prepared using procedure A $(0.09 \mathrm{~g}, 44 \%)$ with imidate 37 and purified using silica gel chromatography (20\% ethyl acetate/80\% hexanes).

37. Reddish solid $(0.09 \mathrm{~g}, 44 \%)$; $\mathrm{mp}=54-56{ }^{\circ} \mathrm{C}$; $\mathrm{TLC} \mathrm{R}_{\mathrm{f}}=0.38$ (20\% ethyl acetate $.80 \%$ hexanes); ${ }^{1} \mathrm{H}$ NMR $\left(400 \mathrm{MHz}, \mathrm{CDCl}_{3}\right) \delta 7.61(\mathrm{dt}, J=8.5,1.9 \mathrm{~Hz}, 2 \mathrm{H}), 7.18(\mathrm{dd}, J=8.5,0.6$ $\mathrm{Hz}, 2 \mathrm{H}), 7.12(\mathrm{dd}, J=1.8,0.8 \mathrm{~Hz}, 1 \mathrm{H}), 6.09(\mathrm{dd}, J=3.2,1.8 \mathrm{~Hz}, 1 \mathrm{H}), 5.89(\mathrm{~d}, J=3.2 \mathrm{~Hz}$, $1 \mathrm{H}), 5.1(\mathrm{~d}, J=8.7 \mathrm{~Hz}, 1 \mathrm{H}), 4.38(\mathrm{q}, J=7.3 \mathrm{~Hz}, 1 \mathrm{H}), 2.37(\mathrm{~s}, 3 \mathrm{H}) .1 .78-1.73(\mathrm{~m}, 2 \mathrm{H})$, 1.28-1.20 (m, 3H), 1.18-1.11 (m, 1H), $0.82(\mathrm{t}, J=7.0 \mathrm{~Hz}, 3 \mathrm{H}) ;{ }^{13} \mathrm{C}$ NMR $(100 \mathrm{MHz}$, $\left.\mathrm{CDCl}_{3}\right) \delta 153.2,142.9,141.7,137.8,129.3,127.0,109.9,106.7,51.7,34.7,27.7,22.1$, $21.4,13.8$.

\section{N-Benzhydryl-4-methylbenzenesulfonamide (39). 41}

Prepared using procedure A $(0.23 \mathrm{~g}, 89 \%)$ using the known imidate ${ }^{42}$ and purified using silica gel chromatography (20\% ethyl acetate/80\% hexanes).

39. White powder $(0.23 \mathrm{~g}, 89 \%) ; \mathrm{mp}=122-124{ }^{\circ} \mathrm{C}$; $\mathrm{TLC} \mathrm{R}_{\mathrm{f}}=0.42$ (20\% ethyl acetate $/ 80 \%$ hexanes); ${ }^{1} \mathrm{H}$ NMR $\left(400 \mathrm{MHz}, \mathrm{CDCl}_{3}\right) \delta 7.56(\mathrm{dt}, J=8.5,2.0 \mathrm{~Hz}, 2 \mathrm{H}), 7.23-7.19(\mathrm{~m}, 6 \mathrm{H})$, 7.15-7.08 (m, 6H), $5.56(\mathrm{~d}, J=6.8 \mathrm{~Hz}, 1 \mathrm{H}), 5.01(\mathrm{~d}, J=6.8 \mathrm{~Hz}, 1 \mathrm{H}), 2.38(\mathrm{~s}, 3 \mathrm{H}) ;{ }^{13} \mathrm{C}$ NMR $\left(100 \mathrm{MHz}, \mathrm{CDCl}_{3}\right) \delta 143.2,140.6,137.4,129.4,128.5,127.5,127.4,127.2,61.4,21.5$.

\section{Methyl 4-(4-(phenyl(2,2,2-trichloro-1-iminoethoxy)methyl)phenyl)butanoate (40)}

To a round bottom flask containing alcohol $\mathbf{5 6}^{25}(0.09 \mathrm{~g}, 0.31 \mathrm{mmol})$ was added DCM (1 $\mathrm{mL})$ followed by trichloroacetonitrile $(0.06 \mathrm{~g}, 0.38 \mathrm{mmol})$ and $\mathrm{DBU}(0.01 \mathrm{~g}, 0.03 \mathrm{mmol})$. The reaction mixture was stirred at room temperature for 4 hours. The solvent was then removed in vacuo. Triethylamine $(1 \mathrm{~mL})$ was added to the residue and the reaction mixture was purified by silica gel chromatography (1\% triethylamine/29\% ethyl acetate/70\% hexanes) to provide $\mathbf{4 0}$ as a pale yellow oil $(0.130 \mathrm{~g}, 99 \%)$.

40. Pale yellow oil ( $0.13 \mathrm{~g}, 99 \%)$; $\mathrm{TLC} \mathrm{R}_{\mathrm{f}}=0.22$ (30\% ethyl acetate $/ 70 \%$ hexanes); IR (KBr) 3651, 3279, 1731, $1495 \mathrm{~cm}^{-1} ;{ }^{1} \mathrm{H}$ NMR $\left(400 \mathrm{MHz} \mathrm{CDCl}_{3}\right) \delta 8.40(\mathrm{br} \mathrm{s}, 1 \mathrm{H})$, 7.43-7.41 (m, 2H), 7.35-7.33 (m, 4H), 7.29-7.28 (m, 1H), $7.15(\mathrm{~d}, J=8.2 \mathrm{~Hz}, 2 \mathrm{H}), 6.92(\mathrm{~s}$, $1 \mathrm{H}), 3.63$ (s, 3H), 2.62 (t, $J=7.4 \mathrm{~Hz}, 2 \mathrm{H}), 2.32$ (t, $J=7.4 \mathrm{~Hz}, 2 \mathrm{H}), 1.93$ (p, $J=7.6 \mathrm{~Hz}$, $2 \mathrm{H}) ;{ }^{13} \mathrm{C}$ NMR $\left(100 \mathrm{MHz}, \mathrm{CDCl}_{3}\right) \delta 173.8,161.4,141.2,139.9,137.5,128.6,128.5,128.0$, 127.1, 126.9, 91.7, 81.3, 51.5, 34.8, 34.4 26.3. Anal. Calcd for $\mathrm{C}_{20} \mathrm{H}_{20} \mathrm{Cl}_{3} \mathrm{NO}_{3}$ : C, 56.03; $\mathrm{H}$, 4.70; N, 3.27. Found: C, 56.28; H, 4.90; N, 3.28.

\section{Methyl 4-(4-((4-methylphenylsulfonamido)(phenyl)methyl)phenyl)butanoate (41)}

Prepared following procedure A $(0.23 \mathrm{~g}, 67 \%)$ with imidate $\mathbf{4 0}$ and purified using silica gel chromatography (30\% ethyl acetate/70\% hexanes) followed by a second purification using silica gel chromatography (100\% DCM). 
41. Clear colorless oil $(0.23 \mathrm{~g}, 67 \%)$; TLC $\mathrm{R}_{\mathrm{f}}=0.45$ (30\% ethyl acetate/70\% hexanes); IR (thin film on KBr) $3328,3227,3062,2950,2864,1731,1599 \mathrm{~cm}^{-1} ;{ }^{1} \mathrm{H}$ NMR $(400 \mathrm{MHz}$, $\left.\mathrm{CDCl}_{3}\right) \delta 7.55(\mathrm{dt}, J=6.6,1.6 \mathrm{~Hz}, 2 \mathrm{H}), 7.20-7.17(\mathrm{~m}, 3 \mathrm{H}), 7.12-7.09(\mathrm{~m}, 4 \mathrm{H}), 7.00(\mathrm{~d}, J=$ $1.6 \mathrm{~Hz}, 4 \mathrm{H}), 5.53(\mathrm{~d}, J=6.9 \mathrm{~Hz}, 1 \mathrm{H}), 5.32(\mathrm{~d}, J=7.2 \mathrm{~Hz}, 1 \mathrm{H}), 3.65$ (s, $3 \mathrm{H}), 2.56$ (t, $J=7.4$ $\mathrm{Hz}, 2 \mathrm{H}), 2.36$ (s, 3H), 2.29 (t, $J=7.4 \mathrm{~Hz}, 2 \mathrm{H}), 1.88(\mathrm{p}, J=7.6 \mathrm{~Hz}, 2 \mathrm{H}) ;{ }^{13} \mathrm{C}$ NMR $(100$ $\left.\mathrm{MHz}, \mathrm{CDCl}_{3}\right) \delta 174.0,143.0,140.8,140.7,138.3,137.4,129.3,128.6,128.5,127.44$, $127.40,127.3,127.2,61.1,51.7,34.6,33.3,26.4,21.4$. Anal. Calcd for $\mathrm{C}_{25} \mathrm{H}_{27} \mathrm{NO}_{4} \mathrm{~S}: \mathrm{C}$, 68.62; H, 6.22; N, 3.20. Found: C, 68.52; H, 6.44; N, 3.59.

\section{$\mathrm{N}$-(4-Methoxybenzyl)-4-methylbenzenesulfonamide (43). ${ }^{43}$}

Prepared using procedure A $(0.17 \mathrm{~g}, 75 \%)$ using the commercially available imidate and purified using silica gel chromatography $(100 \% \mathrm{DCM})$ followed by recrystallization from methanol.

43. White solid $(0.17 \mathrm{~g}, 75 \%) ; \mathrm{mp}=122-123{ }^{\circ} \mathrm{C}$; $\mathrm{TLC} \mathrm{R}_{\mathrm{f}}=0.61(40 \%$ acetone $/ 60 \%$ hexanes); ${ }^{1} \mathrm{H}$ NMR $\left(300 \mathrm{MHz}, \mathrm{CDCl}_{3}\right) \delta 7.75(\mathrm{~d}, J=8.2 \mathrm{~Hz}, 2 \mathrm{H}), 7.31(\mathrm{~d}, J=8.0 \mathrm{~Hz}, 2 \mathrm{H})$, $7.10(\mathrm{~d} J=8.7 \mathrm{~Hz}, 2 \mathrm{H}), 6.79(\mathrm{~d}, J=8.7 \mathrm{~Hz}, 2 \mathrm{H}), 4.59(\mathrm{t}, J=5.8 \mathrm{~Hz}, 1 \mathrm{H}), 4.05(\mathrm{~d}, J=6.0$ $\mathrm{Hz}, 2 \mathrm{H}), 3.78$ (s, 3H), 2.44 (s, 3H); ${ }^{13} \mathrm{C}$ NMR $\left(75 \mathrm{MHz}, \mathrm{CDCl}_{3}\right) \delta$ 159.3, 143.5, 136.9, $129.7,128.3,129.3,127.2,114.1,55.3,46.8,21.5$.

\section{4-Methyl-N-(2-methylbenzyl)benzenesulfonamide (45). ${ }^{44}$}

Prepared using procedure A $(0.12 \mathrm{~g}, 55 \%)$ using the known imidate ${ }^{45}$ and purified using silica gel chromatography (30\% ethyl acetate/70\% hexanes).

45. White solid $(0.12 \mathrm{~g}, 55 \%)$; $\mathrm{mp}=107-109{ }^{\circ} \mathrm{C}$; $\mathrm{TLC} \mathrm{R}_{\mathrm{f}}=0.31$ (30\% ethyl acetate $/ 70 \%$ hexanes); ${ }^{1} \mathrm{NMR}\left(400 \mathrm{MHz}, \mathrm{CDCl}_{3}\right) \delta 7.76(\mathrm{dt}, J=8.5,2.0 \mathrm{~Hz}, 2 \mathrm{H}), 7.31(\mathrm{dd}, J=8.5,0.6$ $\mathrm{Hz}, 2 \mathrm{H}), 7.20-7.10(\mathrm{~m}, 4 \mathrm{H}), 4.45(\mathrm{t}, J=5.8 \mathrm{~Hz}, 1 \mathrm{H}), 4.09(\mathrm{~d}, J=6.0 \mathrm{~Hz}, 2 \mathrm{H}), 2.44(\mathrm{~s}, 3 \mathrm{H})$, $2.24(\mathrm{~s}, 3 \mathrm{H}) ;{ }^{13} \mathrm{C} \mathrm{NMR}\left(100 \mathrm{MHz}, \mathrm{CDCl}_{3}\right) \delta$ 143.6, 136.7, 136.6, 133.9, 130.6, 129.7, $128.9,128.3,127.2,126.2,45.4,21.6,18.8$.

\section{$\mathrm{N}$-(Cyclohex-2-en-1-yl)-4-methylbenzenesulfonamide (49). ${ }^{46}$}

Prepared using procedure A $(0.12 \mathrm{~g}, 60 \%)$ using the known imidate ${ }^{47}$ and purified using silica gel chromatography (30\% ethyl acetate/70\% hexanes).

49. Colorless crystals $(0.12 \mathrm{~g}, 60 \%) ; \mathrm{mp}=99-100{ }^{\circ} \mathrm{C}$; TLC $\mathrm{R}_{\mathrm{f}}=0.45$ (30\% ethyl acetate/70\% hexanes); ${ }^{1} \mathrm{H}$ NMR $\left(400 \mathrm{MHz}, \mathrm{CDCl}_{3}\right) \delta 7.77(\mathrm{dt}, J=8.5,2.0 \mathrm{~Hz}, 2 \mathrm{H}), 7.30$ $(\mathrm{dd}, J=8.5,0.6 \mathrm{~Hz}, 2 \mathrm{H}) .5 .79-5.74(\mathrm{~m}, 1 \mathrm{H}), 5.37-5.32(\mathrm{~m}, 1 \mathrm{H}), 4.44(\mathrm{~d}, J=8.6 \mathrm{~Hz}, 1 \mathrm{H})$, 3.84-3.79 (m, 1H), $2.43(\mathrm{~s}, 3 \mathrm{H}), 2.00-1.87(\mathrm{~m}, 2 \mathrm{H}), 1.79-1.73(\mathrm{~m}, 1 \mathrm{H}), 1.64-1.50(\mathrm{~m}$, $3 \mathrm{H}) ;{ }^{13} \mathrm{C}$ NMR (100 MHz, $\left.\mathrm{CDCl}_{3}\right) \delta$ 143.2, 138.4, 131.5, 129.7, 127.1, 127.0, 49.0, 30.2, $24.5,21.5,19.3$.

\section{N-Allyl-4-methylbenzenesulfonamide (51). ${ }^{48}$}

Prepared using procedure A $(0.05 \mathrm{~g}, 28 \%)$ using the commercially available imidate and purified using silica gel chromatography (30\% ethyl acetate/ $70 \%$ hexanes). 
51. Off-white solid $(0.05 \mathrm{~g}, 28 \%)$; $\mathrm{mp}=53-56{ }^{\circ} \mathrm{C}$; $\mathrm{TLC} \mathrm{R}_{\mathrm{f}}=0.42$ (30\% ethyl acetate $/ 70 \%$ hexanes); ${ }^{1} \mathrm{H}$ NMR (400 MHz CDCl 3 ) $\delta 7.76(\mathrm{dt}, J=8.5,2.0 \mathrm{~Hz}, 2 \mathrm{H}), 7.31(\mathrm{dd}, J=8.5,0.6$ $\mathrm{Hz}, 2 \mathrm{H}), 5.77-5.68(\mathrm{~m}, 1 \mathrm{H}), 5.19-5.08(\mathrm{~m}, 2 \mathrm{H}), 4.51(\mathrm{t}, J=4.50 \mathrm{~Hz}, 1 \mathrm{H}), 3.59$ (tt, $J=6.1$, $1.5 \mathrm{~Hz}, 2 \mathrm{H}), 2.43(\mathrm{~s}, 3 \mathrm{H}) ;{ }^{13} \mathrm{C} \mathrm{NMR}\left(100 \mathrm{MHz}, \mathrm{CDCl}_{3}\right) \delta 143.5,137.0,133.0,129.7,127.2$, $117.7,45.8,21.5$.

\section{$\mathrm{N}, 4$-Dimethylbenzenesulfonamide (53). 49}

Prepared using procedure A $(0.010 \mathrm{~g}, 5 \%)$ using the commercially available imidate and purified using silica gel chromatography (30\% ethyl acetate/70\% hexanes).

53. Off-white solid $(0.010 \mathrm{~g}, 5 \%)$; $\mathrm{mp}=69-71^{\circ} \mathrm{C}$; $\mathrm{TLC} \mathrm{R}_{\mathrm{f}}=0.33$ (30\% ethyl acetate $/ 70 \%$ hexanes); ${ }^{1} \mathrm{H}$ NMR (400 MHz $\left.\mathrm{CDCl}_{3}\right) \delta 7.68(\mathrm{dt}, J=8.3 \mathrm{~Hz}, 2 \mathrm{H}), 7.25(\mathrm{dd}, J=8.4,0.5 \mathrm{~Hz}$, $2 \mathrm{H}), 4.22(\mathrm{~d}, J=4.7 \mathrm{~Hz}, 1 \mathrm{H}), 2.58(\mathrm{~d}, J=5.4 \mathrm{~Hz}, 3 \mathrm{H}), 2.37$ (s, $3 \mathrm{H}) ;{ }^{13} \mathrm{C}$ NMR $(100 \mathrm{MHz}$, $\left.\mathrm{CDCl}_{3}\right) \delta 143.5,135.8,129.7,127.3,29.4,21.5$.

\section{Methyl-4-(4-((4-chlorophenylsulfonamido)(phenyl)methyl)phenyl)butanoate (57). ${ }^{25}$}

Prepared using procedure A $(0.08 \mathrm{~g}, 88 \%)$ and purified using silica gel chromatography (30\% ethyl acetate/70\% hexanes).

57. Clear colorless oil ( $0.08 \mathrm{~g}, 88 \%$ ); TLC $\mathrm{R}_{\mathrm{f}}=0.40$ (30\% ethyl acetate/70\% hexanes); IR (KBr) 3153, 2986, 2820, 1730, $1586 \mathrm{~cm}^{-1}$; ${ }^{1} \mathrm{H}$ NMR (400 MHz CDCl $\left.\mathrm{CD}_{3}\right) \delta .53$ (dt, $J=9.2$, $2.5 \mathrm{~Hz}, 2 \mathrm{H}), 7.25-7.18(\mathrm{~m}, 5 \mathrm{H}), 7.12-7.09(\mathrm{~m}, 2 \mathrm{H}), 7.00$ (br s, $4 \mathrm{H}), 5.58(\mathrm{~d}, J=7.6 \mathrm{~Hz}, 1 \mathrm{H})$, $5.53(\mathrm{~d}, J=7.6 \mathrm{~Hz}, 1 \mathrm{H}), 3.65(\mathrm{~s}, 3 \mathrm{H}), 2.57(\mathrm{t}, J=7.4 \mathrm{~Hz}, 2 \mathrm{H}), 2.30(\mathrm{t}, J=7.4 \mathrm{~Hz}, 2 \mathrm{H}), 1.89$ $(\mathrm{p}, J=7.6 \mathrm{~Hz}, 2 \mathrm{H}) ;{ }^{13} \mathrm{C} \mathrm{NMR}\left(100 \mathrm{MHz}, \mathrm{CDCl}_{3}\right) \delta 173.9,141.1,140.2,139.0,138.7$, 137.7, 128.9, 128.7, 128.59, 128.57, 127.7, 127.4, 127.3, 61.3, 51.6, 34.6, 33.4, 26.4. Anal. Calcd for $\mathrm{C}_{24} \mathrm{H}_{24} \mathrm{ClNO}_{4} \mathrm{~S}$ : C, 62.94; H, 5.28; N, 3.06. Found: C, 63.17; H, 5.35; N, 3.22.

\section{Supplementary Material}

Refer to Web version on PubMed Central for supplementary material.

\section{Acknowledgements}

Financial support was provided by the National Institute of General Medical Sciences (R15-GM116054). Acknowledgement is also made to the Donors of the American Chemical Society Petroleum Research Fund for a New Directions award in support of this research (54823-ND1). NMR spectra were obtained at Syracuse University using instrumentation acquired with the assistance of the National Science Foundation (CHE-1229345).

\section{References}

1. (a) Shah SSA, Rivera G, Ashfaq M. Mini-Rev. Med. Chem. 2013; 13:70. [PubMed: 22625411] (b) Scozzafava A, Owa T, Mastrolorenzo A, Supuran CT. Curr. Med. Chem. 2003; 10:925. [PubMed: 12678681] (c) Supuran CT, Casini A, Scozzafava A. Med. Res. Rev. 2003; 23:535. [PubMed: 12789686]

2. (a) McGrath NA, Brichacek M, Njardarson JT. J. Chem. Educ. 2010; 87:1348.(b) Ilardi EA, Vitaku E, Njardarson JT. J. Med. Chem. 2014; 57:2832. [PubMed: 24102067]

3. Flick AC, Ding HX, Leverett CA, Kyne REJ, Liu KK, Fink SJ, O’Donnell CJ. Bioorg. Med. Chem. 2016; 24:1937. [PubMed: 27020685] 
4. (a) Hewawasam P, Tu Y, Gao M, Hanumegowda U, Knipe J, Lemm JA, Parker DD, Rigat KL, Roberts SB, Meanwell NA, Kadow JF. Bioorg. Med. Chem. Lett. 2016; 26:936. [PubMed: 26747393] (b) Chupak LS, Zheng X, Hu S, Huang Y, Ding M, Lewis MA, Westphal RS, Blat Y, McClure A, Gentles RG. Bioorg. Med. Chem. 2016; 24:1455. [PubMed: 26917221] (c) Pardeshi KA, Malwal SR, Banerjee A, Lahiri S, Rangarajan R, Chakrapani H. Bioorg. Med. Chem. Lett. 2015; 25:2694. [PubMed: 25981687] (d) Eminoglu A, Vullo D, Asik A, Colak DN, Canakci S, Belduz AO, Supuran CT. Bioorg. Med. Chem. Lett. 2016; 26:1821. [PubMed: 26920803] (e) Schroeder GM, Wei D, Banfi P, Cai Z-W, Lippy J, Menichincheri M, Modugno M, Naglich J, Penhallow B, Perez HL, Sack J, Schmidt RJ, Tebben A, Yan C, Zhang L, Galvani A, Lombardo LJ, Borzilleri RM. Bioorg. Med. Chem. Lett. 2012; 22:3951. [PubMed: 22608393]

5. (a) Kleschick WA, Gerwick BC, Carson CM, Monte WT, Snider SW. J. Agric. Food Chem. 1992; 40:1083.(b) Hultgren RP, Hudson RJM, Sims GK. J. Agric. Food Chem. 2002; 50:3236. [PubMed: 12009993] (c) Grossman MR, Mispagel ME, Bowen JM. J. Agric. Food Chem. 1992; 40:2505.

6. (a) Ji S, Gortler LB, Waring A, Battisti AJ, Bank S, Closson WD, Wriede PA. J. Am. Chem. Soc. 1967; 89:5311.(b) Nishimura T, Yasuhara Y, Hayashi T. Org. Lett. 2006; 8:979. [PubMed: 16494489]

7. (a) Weinreb SM, Demko DM, Lessen TA, Demers JP. Tetrahedron Lett. 1986; 27:2099.(b) Henry JR, Marcin LR, McIntosh MC, Scola PM, Davis Harris G Jr, Weinreb SM. Tetrahedron Lett. 1989; 30:5709.(c) Ribière P, Declerck V, Martinez J, Lamaty F. Chem. Rev. 2006; 106:2249. [PubMed: 16771449]

8. (a) Fukuyama T, Jow C-K, Cheung M. Tetrahedron Lett. 1995; 36:6373.(b) Kan T, Fukuyama T. Chem. Commun. 2004:353.

9. (a) Kamal A, Reddy JS, Bharathi EV, Dastagiri D. Tetrahedron Lett. 2008; 49:348.(b) Lakrout S, K'Tir H, Amira A, Berredjem M, Aouf N-E. RSC Adv. 2014; 4:16027.

10. (a) Marcotullio MC, Campagna V, Sternativo S, Costantino F, Curini M. Synthesis. 2006:2760.(b) Adib M, Sheikhi E, Moghaddam GS, Bijanzadeh HR. Tetrahedron Lett. 2010; 51:5646.(c) Rad MNS, Behrouz S. Mol. Diversity. 2013; 17:745.(d) Yu TT, Qi L-J, Cui D-M, Zhang C, Zhao Y. Bull. Chem. Soc. Jpn. 2015; 88:610.

11. (a) Gellert BA, Kahlcke N, Feurer M, Roth S. Chem. Eur. J. 2011; 17:12203. [PubMed: 21922582] (b) Abdel-Magid AF, Carson KG, Harris BD, Maryanoff CA, Shah RD. J. Org. Chem. 1996; 61:3849. [PubMed: 11667239] (c) Abdel-Magid AF, Mehrman SJ. Org. Process Res. Dev. 2006; 10:971.(d) Baxter EW, Reitz AB. Org. React. 2002; 59:1.

12. Kelleher F, Proinsias K. ó. Tetrahedron Lett. 2007; 48:4879.

13. (a) Karshtedt D, Bell AT, Tilley TD. J. Am. Chem. Soc. 2005; 127:12640. [PubMed: 16144412] (b) Manzoni MR, Zabawa TP, Kasi D, Chemler SR. Organometallics. 2004; 23:5618.(c) Paderes MC, Chemler SR. Org. Lett. 2009; 11:1915. [PubMed: 19331361] (d) Sequeira FC, Turnpenny BW, Chemler SR. Angew. Chem., Int. Ed. 2010; 49:6365.(e) Turnpenny BW, Chemler SR. Chem. Sci. 2014; 5:1786. [PubMed: 24932404] (f) Sevov CS, Zhou J, Hartwig JF. J. Am. Chem. Soc. 2012; 134:11960. [PubMed: 22780090] (g) Huang L, Arndt M, Goossen K, Heydt H, Goossen LJ. Chem. Rev. 2015; 115:2596. [PubMed: 25721762] (h) Kanno O, Kuriyama W, Wang ZJ, Toste FD. Angew. Chem., Int. Ed. 2011; 50:9919.(i) Severin R, Doye S. Chem. Soc. Rev. 2007; 36:1407. [PubMed: 17660874]

14. (a) Pham MV, Ye B, Cramer N. Angew. Chem., Int. Ed. 2012; 51:10610.(b) Archambeau A, Rovis T. Angew. Chem., Int. Ed. 2015; 54:13337.(c) Chu L, Wang X-C, Moore CE, Rheingold AL, Yu JQ. J. Am. Chem. Soc. 2013; 135:16344. [PubMed: 24151991] (d) Roizen JL, Zalatan DN, Du Bois J. Angew. Chem., Int. Ed. 2013; 52:11343.(e) Roizen JL, Harvey ME, Du Bois J. Acc. Chem. Res. 2012; 45:911. [PubMed: 22546004] (f) Sreedhar B, Ravi V, Yada D. Tetrahedron Lett. 2011; 52:1208.

15. (a) Hayashi T, Ishigedani M. J. Am. Chem. Soc. 2000; 122:976.(b) Duan H-F, Jia Y-X, Wang L-X, Zhou Q-L. Org. Lett. 2006; 8:2567. [PubMed: 16737315] (c) Luo Y, Wu J. Chem. Commun. 2010; 46:3785.(d) Beisel T, Manolikakes G. Org. Lett. 2015; 17:3162. [PubMed: 26053313] (e) Li Y, Li B-J, Wang W-H, Huang W-P, Zhang X-S, Chen K, Shi Z-J. Angew. Chem., Int. Ed. 2011; 50:2115. (f) Hesp KD, Bergman RG, Ellman JA. Org. Lett. 2012; 14:2304. [PubMed: 22500854]

J Org Chem. Author manuscript; available in PMC 2017 September 02. 
16. (a) von Matt P, Loiseleur O, Koch G, Pfaltz A, Lefeber C, Feucht T, Helmchen G. Tetrahedron: Asymmetry. 1994; 5:573.(b) Jumnah R, Williams JMJ, Williams AC. Tetrahedron Lett. 1993; 34:6619.(c) Maity AK, Chatterjee PN, Roy S. Tetrahedron. 2013; 69:942.

17. (a) Saidi O, Blacker AJ, Lamb GW, Marsden SP, Taylor JE, Williams JMJ. Org. Process Res. Dev. 2010; 14:1046.(b) Martínez-Asencio A, Yus M, Ramón DJ. Synthesis. 2011:3730.(c) Garcia Ruano JL, Parra A, Aleman J, Yuste F, Mastranzo VM. Chem. Commun. 2009:404.(d) MartinezAsencio A, Yus M, Ramon DJ. Synthesis. 2011:3730.(e) Guillena G, Ramon DJ, Yus M. Chem. Rev. 2010; 110:1611. [PubMed: 19928825]

18. (a) Shi W, Bai C-M, Zhu K, Cui D-M, Zhang C. Tetrahedron. 2014; 70:434.(b) Trillo P, Baeza A, Najera C. ChemCatChem. 2013; 5:1538.

19. (a) Adhikari AA, Shah JP, Howard KT, Russo CM, Wallach DR, Linaburg MR, Chisholm JD. Synlett. 2014:283.(b) Shah JP, Russo CM, Howard KT, Chisholm JD. Tetrahedron Lett. 2014; 55:1740.(c) Howard KT, Duffy BC, Linaburg MR, Chisholm JD. Org. Biomol. Chem. 2016; 14:1623. [PubMed: 26691695] (d) Duffy BC, Howard KT, Chisholm JD. Tetrahedron Lett. 2015; 56:3301.(e) Wallach DR, Stege PC, Shah JP, Chisholm JD. J. Org. Chem. 2015; 80:1993. [PubMed: 25568933]

20. (a) Iversen T, Bundle DR. J. Chem. Soc., Chem. Commun. 1981:1240.(b) Wessel H-P, Iversen T, Bundle DR. J. Chem. Soc. Pekin 1. 1985:2247.

21. (a) Schmidt RR, Michel J. Angew. Chem. 1980; 92:763.(b) Schmidt RR, Michel J. J. Carbohydr. Chem. 1985; 4:141.(c) Schmidt, RR.; Jung, K-H. Preparative Carbohydrate Chemistry. Hanessian, S., editor. CRC Press; 1997. p. 283

22. Kuroda Y, Harada S, Oonishi A, Yamaoka Y, Yamada K.-i. Takasu K. Angew. Chem. Int. Ed. 2015; 54:8263.

23. Ragnarsson U, Grehn L. Acc. Chem. Res. 1991; 24:285.

24. Poss MA, Reid JA. Tetrahedron Lett. 1992; 33:7291.

25. Sakurai S, Ogawa N, Suzuki T, Kato K.-i. Ohashi T, Yasuda S, Kato H, Ito Y. Chem. Pharm. Bull. 1996; 44:765. [PubMed: 8681409]

26. (a) Sakurai S, Ogawa N, Suzuki T, Kato K.-i. Ohashi T, Yasuda S, Kato H. Chem. Pharm. Bull. 1996; 44:1510. [PubMed: 8795270] (b) Sakurai S, Ogawa N, Onogi Y, Takeshita M, Takahashi H, Ohashi T, Kato K-I, Yasuda S, Kato H. Chem. Pharm. Bull. 1997; 45:849. [PubMed: 9178522]

27. Giner X, Najera C. Org. Lett. 2008; 10:2919. [PubMed: 18553970]

28. Wang L, Zhou Q, Qu C, Wang Q, Cun L, Zhu J, Deng J. Tetrahedron. 2013; 69:6500.

29. Zotto CD, Michaux J, Zarate-Ruiz A, Gayon E, Virieux D, Campagne J-M, Terrasson V, Pieters G, Gaucher A, Prim D. J. Organomet. Chem. 2011; 696:296.

30. Wang Z, Zhang Y, Fu H, Jiang Y, Zhao Y. Org. Lett. 2008; 10:1863. [PubMed: 18386905]

31. Fiori KW, Du Bois J. J. Am. Chem. Soc. 2007; 129:562. [PubMed: 17227019]

32. Deeming AS, Russell CJ, Willis MC. Angew. Chem. Int. Ed. 2015; 54:1168.

33. Nishioka Y, Uchida T, Katsuki T. Angew. Chem. Int. Ed. 2013; 52:1739.

34. Robinson RI, Fryatt R, Wilson C, Woodward S. Eur. J. Org. Chem. 2006:4483.

35. Yang C-H, Fan W-W, Liu G-Q, Duan L, Li L, Li Y-M. RSC Adv. 2015; 5:61081.

36. Yadav JS, Subba Reddy BV, Jain R, Baishya G. Tetrahedron Lett. 2008; 49:3015.

37. Fan X, Fu L-A, Li N, Lv H, Cui X-M, Qi Y. Org. Biomol. Chem. 2013; 11:2147. [PubMed: 23380886]

38. Swenton JS, Callinan A, Wang S. J. Org. Chem. 1992; 57:78.

39. Kazancioglu EA, Kazancioglu MZ, Fistikci M, Secen H, Altundas R. Org. Lett. 2013; 15:4790. [PubMed: 24001310]

40. Zhou W-S, Lu Z-H, Wang Z-M. Tetrahedron. 1993; 49:2641.

41. Georgy M, Boucard V, Debleds O, Zotto CD, Campagne J-M. Tetrahedron. 2009; 65:1758.

42. Ali IAI, El Ashry ESH, Schmidt RR. Eur. J. Org. Chem. 2003:4121.

43. Molander GA, Fleury-Brégeot N, Hiebel M-A. Org. Lett. 2011; 13:1694. [PubMed: 21366299]

44. Müther K, Mohr J, Oestreich M. Organometallics. 2013; 32:6643.

45. Li C, Li W, Wang J. Tetrahedron Lett. 2009; 50:2533.

J Org Chem. Author manuscript; available in PMC 2017 September 02. 
46. Xu X, Wu H, Li Z, Sun X, Wang Z. Tetrahedron. 2015; 71:5254.

47. Overman LE. J. Am. Chem. Soc. 1976; 98:2901.

48. Kobayashi Y, Inukai S, Kondo N, Watanabe T, Sugiyama Y, Hamamoto H, Shioiri T, Matsugi M. Tetrahedron Lett. 2015; 56:1363.

49. Laha JK, Sharma S, Dayal N. Eur. J. Org. Chem. 2015:7885. 
<smiles>N=C(Cl)C(Cl)Cl</smiles>

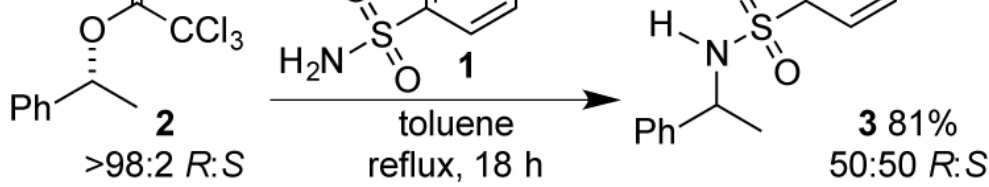

Scheme 1.

Substitution with a Chiral Trichloroacetimidate. 

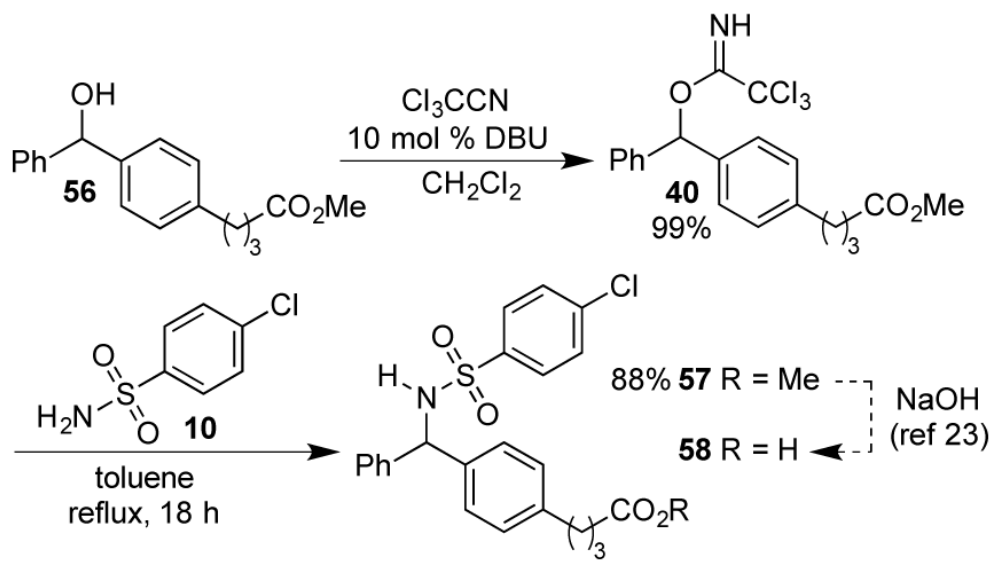

Scheme 2.

Synthesis of a Ketoprofen Analog. 
Table 1

Addition of Toluenesulfonamide 1 to Phenethyl Trichloroacetimidate 2.

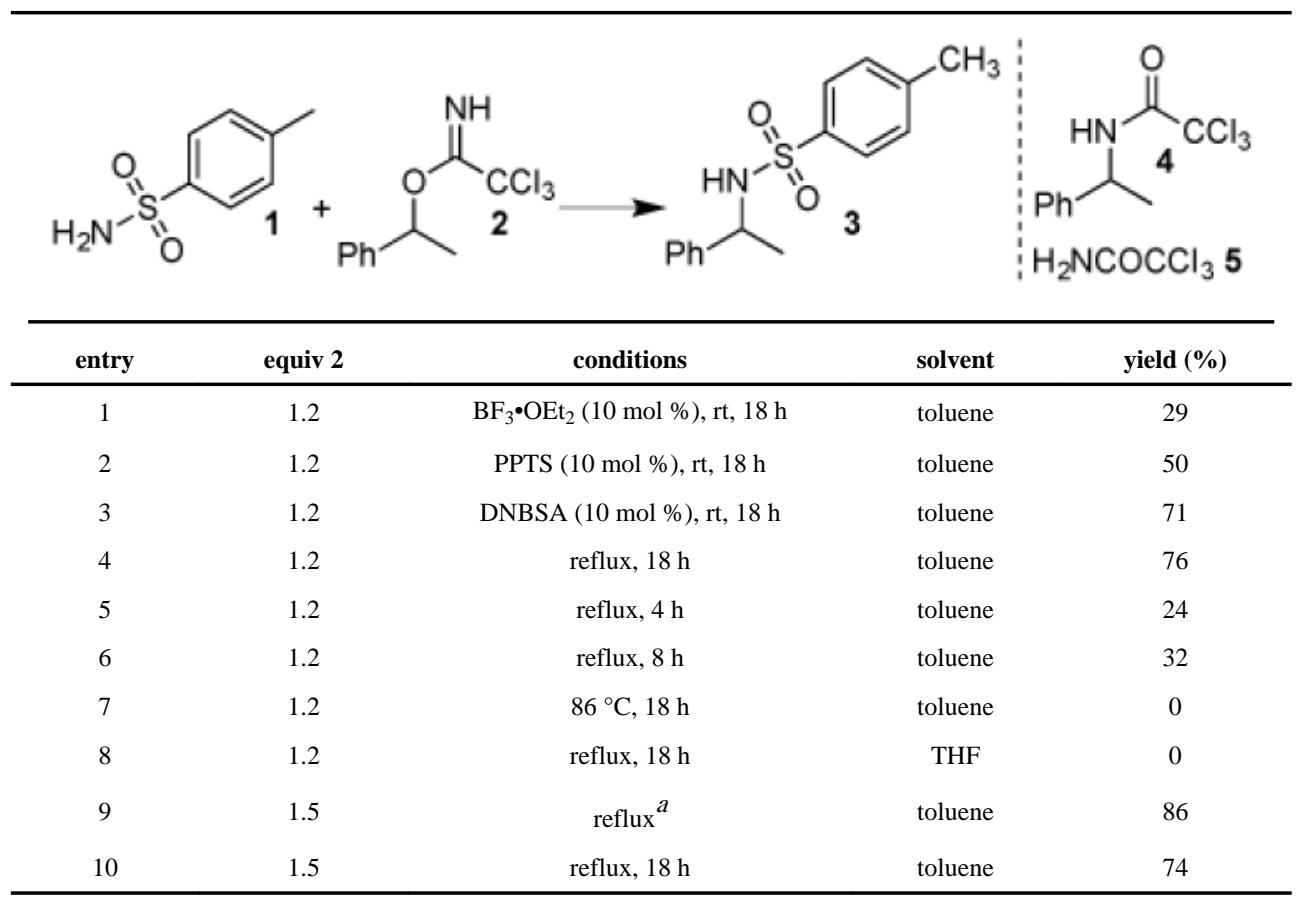

${ }^{a}$ The imidate added in 6 portions (one every $30 \mathrm{~min}$ ) over $2.5 \mathrm{~h}$, then the reaction mixture was refluxed for another $16 \mathrm{~h}$. 


\section{Table 2}

Alkylation of Sulfonamides with Imidate 2.

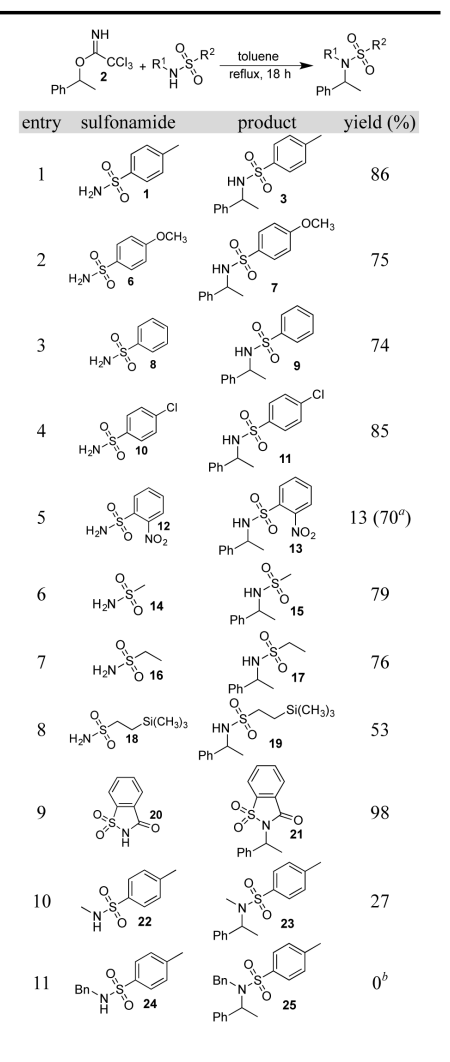

${ }^{a}$ Yield for a modified procedure using $10 \mathrm{~mol} \% \mathrm{BF}_{3} \cdot \mathrm{OEt}_{2}$ in toluene at room temperature with the imidate being added as a refluxing solution of sulfonamide with a syringe pump over 1 hour.

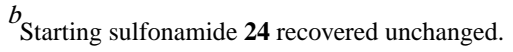


Table 3

Addition of $p$-Toluenesulfonamide 1 to Different Trichloroacetimidates.

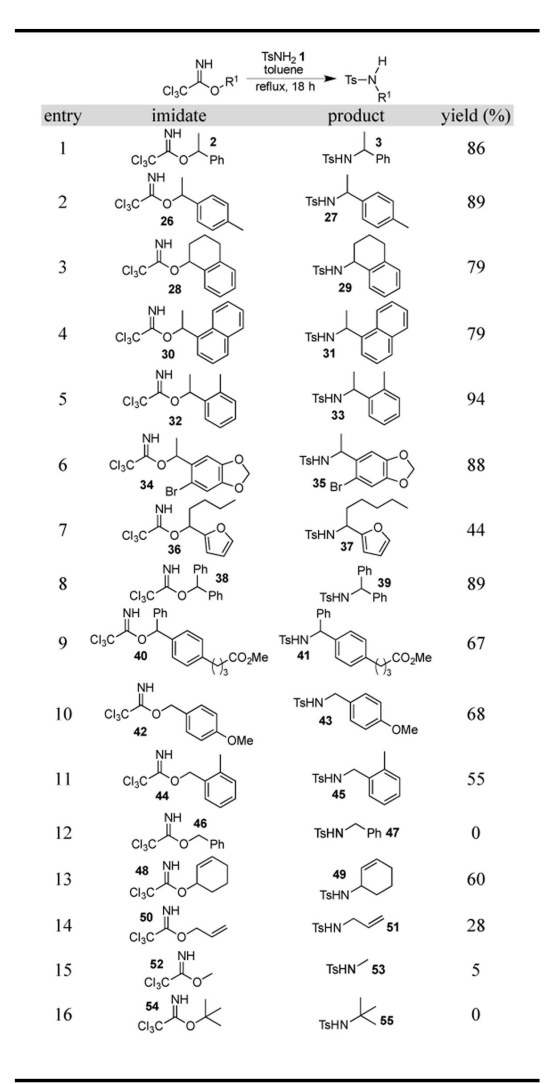

롤

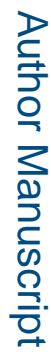

J Org Chem. Author manuscript; available in PMC 2017 September 02. 